\title{
Elektorale Koordination, legislative Kohäsion und der Aufstieg der modernen Massenpartei: Die Grenzen des Mehrheitswahlrechts im Deutschen Kaiserreich, 1890-1918'
}

\author{
Valentin Schröder / Philip Manow
}

Electoral Coordination, Legislative Cohesion, and the Rise of the Modern Mass Party: The Limits of Majoritarian Representation in Germany, 1890-1918

Abstract: Explanations of the switch from majoritarian (MR) to proportional representation (PR) at the beginning of the $20^{\text {th }}$ century focus on parties' expected gains of seats. But these gains had actually been achieved even under MR, via local-level electoral alliances. Moreover, the underlying assumption of major parties of the time as unitary actors appears inaccurate. Rather, PR proved attractive for leaders of established parties whose struggle for control over backbencher behavior was exacerbated by increasing alliance diversity and the rise of modern mass parties. This rise, incarnated in the socialist parties, thus threatened legislative, not electoral majorities of established parties. Using encompassing datasets on alliances and parliamentary voting behavior for Germany in the period 1890-1918, we show this argument to hold empirically.

\section{Einleitung}

In der Politikwissenschaft hat das Interesse an den Wahlrechtsreformen im frühen 20. Jahrhundert sprunghaft zugenommen (Blais et al. 2004; Ahmed 2010; Kreuzer 2010; Cusack et al. 2007, 2010; Boix 1999; Ahmed 2012; Calvo 2009; Mares u. Leemann 2014). Angesichts der substanziellen Auswirkungen dieser frühen Weichenstellungen ist dieses gesteigerte Interesse auch gut begründet (vgl. etwa Iversen u. Soskice 2006; Gourevitch u. Shinn 2007; Powell 2000). Die bisherige Debatte leidet jedoch darunter, dass wir aufgrund einer unzureichenden Datengrundlage ein bislang nur sehr begrenztes Verständnis von der Funktionsweise der absoluten Mehrheitswahl (Majority Representation, MR) haben, als demjenigen Wahlsystem, das der Einführung der Verhältniswahl (Proportional Representation, PR) in den meisten europäischen Ländern voranging (Blais et al. 2004). Doch je weniger wir die Effekte der absoluten Mehrheitswahl verstehen, umso schwerer muss es uns fallen, die Einführung der Verhältniswahl zu erklären.

Nehmen wir zum Beispiel die These, dass mit dem Wechsel zur Verhältniswahl die parlamentarische Abstimmungskohäsion entscheidend anstieg, sodass erst

1 Wir möchten Carsten Nickel für seine wertvolle Mitarbeit im ,Bremen Reichstag-Projekt` (http:// www.zes.uni-bremen.de/das-zentrum/organisation/mitglieder/philip-manow/projekte/?proj=7) ganz herzlich danken. Vorherige Versionen des Aufsatzes wurden vorgestellt am Institut für Höhere Studien, Wien, am Juan-March Institut, Madrid, sowie an der Universität Potsdam. Wir danken insbesondere Michael Koß, Ronald Rogowski und Christian Stecker sowie zwei anonymen Gutachtern für außerordentlich hilfreiche Kommentare. Wir möchten uns auch bei Marlon Brandt und Volker Lindhauer für hervorragende Mithilfe bei der Recherche und Codierung der verwendeten Daten bedanken. 
jetzt Parteien in einer Zeit der Etablierung „nationaler Kapitalismen“ kohäsive Vertreter gesellschaftlicher Interessenlagen werden konnten (Cusack et al. 2010). Angesichts des britischen Falls stellt sich sofort die Frage, ob für die Herstellung von Fraktionsdisziplin tatsächlich die Einführung der PR unumgänglich war (Cox 1987). Zudem: Wir wissen, dass die Abstimmungskohäsion nach 1918 im Deutschen Reichstag relativ hoch war (Lehmann 2010), aber war sie zuvor so viel niedriger? Ein weiteres Beispiel ist Stein Rokkans berühmte Erklärung für den Siegeszug der Verhältniswahl, der zufolge Schwierigkeiten beim Schmieden von Wahlbündnissen ein entscheidendes Motiv für Parteien des bürgerlich-konservativen Spektrums darstellen, sich angesichts der elektoralen Bedrohung durch die eingangs des 20. Jahrhunderts drastisch erstarkenden sozialistischen Parteien vom Mehrheitswahlrecht abzuwenden (Rokkan 1970; Boix 1999). Aber war es tatsächlich so schwierig, Wahlbündnisse einzugehen, sodass die „etablierten Parteien“, also die Verfechter des konstitutionellen Status quo, unter der MR der „Bedrohung von Links“ (Boix 1999) hilflos ausgeliefert waren? Diese für die Debatte über die Gründe für die Einführung der PR zentralen Fragen können wir bislang aufgrund fehlender Daten nicht befriedigend beantworten.

Die Funktionsweise der MR lässt sich für das Deutsche Kaiserreich besonders gut nachvollziehen, denn dieser Wahlmodus kam über den gesamten Zeitraum von 1867 bis 1918 zur Anwendung, also in einer Zeit fundamentalen sozio-ökonomischen Wandels und unter einer zwar umstrittenen, aber vollentwickelten Verfassungsordnung. Besonders die starke Rolle der deutschen Sozialdemokratie bei gleichzeitig deutlicher Fragmentierung des etablierten Parteienspektrums bietet zudem eine hervorragende Gelegenheit zur Überprüfung von Rokkans Koordinierungsthese. Deswegen steht der deutsche Fall als exemplarischer Fall für die Wirkungsweise der Mehrheitswahl im Zentrum unserer folgenden Analyse.

Für sie haben wir sowohl die Parteienbündnisse auf Wahlkreisebene als auch alle 552 namentlichen Abstimmungen im Reichstag für den Zeitraum 1890-1918 kodiert. Auf der Grundlage dieser Daten entwickeln wir im Folgenden ein Argument, das nach unserem Kenntnisstand in der Wahlrechtsreformdebatte noch nicht formuliert wurde. Bislang werden die Parteipositionen vor allem mit den von den Parteien erwarteten Mandatsgewinnen bzw. -verlusten erklärt, d. h. mit Fokus auf die elektoralen Reformeffekte. Im Gegensatz zur vorherrschenden Literatur zeigen wir jedoch, dass die elektorale Koordination unter der MR weitgehend unproblematisch und damit auch das Ziel der Mandatsmaximierung nicht grundsätzlich gefährdet war. Doch während die etablierten Parteien durch Wahlkreisabsprachen durchaus in der Lage waren, „ihre“ Stimmen in Mandate zu übersetzen, sahen sie sich vor erhebliche Probleme gestellt, diese Mandate auch in legislative Mehrheiten zu transformieren. Wir argumentieren also, dass die Vertreter der nicht-sozialistischen Parteien unter dem Mehrheitswahlrecht mit den legislativen Folgen erfolgreicher und weitverbreiteter elektoraler Koordination zu kämpfen hatten, nicht aber mit dieser Koordination selbst.

Im Folgenden präsentieren wir zunächst unser mikro-fundiertes Argument, indem wir an Stelle der analytischen Einheit „Partei“ zwei Arten von Akteuren unterscheiden: Parteieliten und Abgeordnete bzw. „Frontleute und Hinterbänkler“ (Abschnitt 2). Anschließend testen wir Rokkans These, indem wir die Bildung von 
Wahlbündnissen im Vorfeld von Reichstagswahlen untersuchen (Abschnitt 3). Danach überprüfen wir die legislativen Effekte elektoraler Koordination unter dem Mehrheitswahlrecht (Abschnitt 4). Unser Argument lautet, dass die - lokal definierten - Anreizmechanismen des Mehrheitswahlrechts der Herstellung parlamentarischer Abstimmungskohäsion entgegenstanden. Das impliziert, dass die einfachen Abgeordneten der etablierten Parteien jeder Wahlrechtsreform ihre $\mathrm{Zu}$ stimmung verweigern sollten, die diesen lokal geprägten Anreizen nicht entsprach. Nun kam es aber im Kaiserreich zu einer Wahlreform, und zwar zwischen April 1917 und Juli 1918 (also noch vor der Einführung der PR in der Novemberrevolution). Diese Reform dient uns daher als Testfall für unser Argument. Wir werden sie in Abschnitt 5 genauer betrachten. Die Generalisierbarkeit unseres Arguments über den deutschen Fall hinaus ist Gegenstand unserer Konklusion (Abschnitt 6).

\section{Elektorale vs. legislative Anreize: die parlamentarischen Effekte des Mehrheitswahlrechts}

Die Literatur zu Wahlreformen folgt meist dem ebenso vertrauten wie einleuchtenden „cui bono?“-Erklärungsmuster. Das „bono“ bezieht sich dabei in der Regel auf die simple Logik der Mandatsmaximierung, während das „cui“ Parteien als Akteure in den Mittelpunkt stellt, die durch die jeweilige Reform Sitzgewinn erhoffen oder Sitzverlust befürchten (Boix 1999; Rokkan 1970; Benoit 2004; Blais et al. 2004; Calvo 2009). Auf einer noch höheren Abstraktionsebene ist der Vorschlag angesiedelt, für die Einführung der PR in Westeuropa zwei politische Lager zu unterscheiden, nämlich die etablierten Parteien und die nicht-etablierten Parteien als ihre Gegner (Boix 1999). Doch bilden die Parteien die Untersuchungseinheit, so verliert man u. E. die entscheidende Konfliktdimension aus dem Blick. In diesem standen sich Parteiführer und ihre Anhänger innerhalb der Parteien gegenüber, was sich in den Konflikt zwischen den Befürwortern des Typus der neuen Massenpartei und denen des alten Typus der Honoratiorenpartei übersetzte (Katz u. Mair 1995). Herkömmliche Erklärungen setzen somit analytisch oft genau jene Frage als geklärt voraus, die im Zentrum der damaligen Debatte stand: Sollten sich politische Parteien zu zentralisierten, kohärenten, korporativen, intern verpflichtungsfähigen Akteuren entwickeln, sodass Wähler fortan für Parteien anstatt für Kandidaten stimmen würden (Cox 1987), sollte der Mechanismus demokratischer Verantwortlichkeit damit zukünftig ein kollektiver statt ein individueller sein (Carey 2009)? Genau auf diese Frage stellte Robert Michels' Kritik der Herausbildung einer neuen „Oligarchie“ ab (Michels [1911] 1957). Es erscheint uns bezeichnend, dass eben jener zeitgenössische Schlüsselbegriff der Parteioligarchie in keinem der zahlreichen neueren Erklärungsansätze zur Einführung der Verhältniswahl eine Rolle zu spielen scheint. 
Tabelle 1: Wahlsysteme 1900-1925 in den europäischen Staaten mit mindestens allgemeinem direktem Männerwahlrecht spätestens im Jahr 1919 (Kategorisierung nach Carey u. Hix 2006).

\begin{tabular}{|c|c|c|c|c|c|c|c|}
\hline & \multicolumn{6}{|c|}{ Wahlsystem } & \multirow{2}{*}{$\begin{array}{l}\text { All- } \\
\text { gemeines } \\
\text { Wahlrecht }\end{array}$} \\
\hline & 1900 & 1905 & 1910 & 1915 & 1920 & 1925 & \\
\hline Österreich & (Kurien) & (Kurien) & $\mathrm{MR}>\mathrm{MR}$ & $\mathrm{MR}>\mathrm{MR}$ & PR & PR & 1907 \\
\hline Belgien & EA & EA & EA & EA & PR & PR & 1919 \\
\hline $\begin{array}{l}\text { Tschecho- } \\
\text { slowakei }\end{array}$ & - & - & - & - & PR & PR & 1918 \\
\hline Dänemark & PL & PL & PL & $\mathrm{PR}+\mathrm{PL}$ & PR & PR & 1915 \\
\hline Estland & - & - & - & - & PR & PR & 1919 \\
\hline Finnland & - & - & - & - & PR & PR & 1907 \\
\hline Frankreich & $\mathrm{MR}>\mathrm{PL}$ & $\mathrm{MR}>\mathrm{PL}$ & $\mathrm{MR}>\mathrm{PL}$ & $\mathrm{MR}>\mathrm{PL}$ & PR* & $\mathrm{PR}^{*}$ & 1848 \\
\hline Deutschland & $\mathrm{MR}>\mathrm{MR}$ & $\mathrm{MR}>\mathrm{MR}$ & $\mathrm{MR}>\mathrm{MR}$ & $\mathrm{MR}>\mathrm{MR}$ & PR & PR & 1867 \\
\hline Griechenland & $\mathrm{MR}>\mathrm{MR}$ & $\mathrm{MR}>\mathrm{MR}$ & $\mathrm{MR}>\mathrm{MR}$ & $\mathrm{MR}>\mathrm{MR}$ & $\mathrm{MR}>\mathrm{MR}$ & PR & 1864 \\
\hline Großbritannien & PL & PL & PL & PL & PL & PL & 1918 \\
\hline Island & - & - & - & - & PR & PR & 1915 \\
\hline Irland & - & - & - & - & - & $P R * *$ & 1918 \\
\hline Italien & $\mathrm{MR}>\mathrm{MR}$ & $\mathrm{MR}>\mathrm{MR}$ & $\mathrm{MR}>\mathrm{MR}$ & $\mathrm{MR}>\mathrm{MR}$ & PR & PR & 1913 \\
\hline Luxemburg & $\mathrm{MR}>\mathrm{PL}$ & $\mathrm{MR}>\mathrm{PL}$ & $\mathrm{MR}>\mathrm{PL}$ & $\mathrm{MR}>\mathrm{PL}$ & PR & PR & 1918 \\
\hline Niederlande & $\mathrm{MR}>\mathrm{MR}$ & $\mathrm{MR}>\mathrm{MR}$ & $\mathrm{MR}>\mathrm{MR}$ & $\mathrm{MR}>\mathrm{MR}$ & PR & PR & 1917 \\
\hline Norwegen & - & $\mathrm{MR}>\mathrm{PL}$ & $\mathrm{MR}>\mathrm{PL}$ & $\mathrm{MR}>\mathrm{PL}$ & PR & PR & 1905 \\
\hline Polen & - & - & - & - & PR & PR & 1919 \\
\hline Portugal & PL & PL & PL & PL & PL & PL & $(1918-19)$ \\
\hline Rumänien & $\mathrm{MR}>\mathrm{PL}$ & $\mathrm{MR}>\mathrm{PL}$ & $\mathrm{MR}>\mathrm{PL}$ & $\mathrm{MR}>\mathrm{PL}$ & PR & PR & 1918 \\
\hline Spanien & PL & PL & PL & PL & PL & PL & 1890 \\
\hline Schweden & PL & PL & PR & PR & PR & PR & 1907 \\
\hline Schweiz & $\mathrm{MR}>\mathrm{MR}>\mathrm{PL}$ & $\mathrm{MR}>\mathrm{MR}>\mathrm{PI}$ & $\mathrm{MR}>\mathrm{MR}>\mathrm{PI}$ & $M R>M R>P L$ & $\mathrm{PR}+\mathrm{PL}$ & $\mathrm{PR}+\mathrm{PL}$ & 1848 \\
\hline
\end{tabular}

Erläuterungen: Allgemeines Wahlrecht: Jahr der Einführung des allgemeinen direkten Wahlrechts für Männer; Wahlsysteme: PR: Verhältniswahl; MR: Absolute Mehrheitswahl; PL: Relative Mehrheitswahl; PR+PL: Verhältniswahlkomponente nur wirksam in Abhängigkeit von entsprechender Magnitude (mindestens Zwei) des Wahlkreises; EA: Wahlsystem eigener Art (durchschnittliche Magnitude <4); $P^{*}$ : PR in kleinen Wahlkreisen (durchschnittliche Magnitude 5-7), mit allen Mandaten des Wahlkreises für eine Liste, wenn sie dort die absolute Mehrheit der Stimmen auf sich vereint; PR**: Präferenzwahlsystem in großen Wahlkreisen (durchschnittliche Magnitude >7); ,”“: trennt bei Wahlsystemen mit mehr als einem möglichen Wahlgang die genannten Voraussetzungen für den Mandatserwerb nach Runden; Fett: PR erstmals in Kraft.

Sonstige europäische Staaten, in denen bis 1919 kein allgemeines Wahlrecht bestand: Nach der Unabhängigkeit 1919 wurde das allgemeine Wahlrecht in Lettland, Litauen und Ungarn im Jahr 1920 eingeführt. Während das serbische Parlament in unseren Quellen nicht als vom Volk gewählt eingeordnet wird, wurden in Jugoslawien allgemeines Männerwahlrecht und PR im Jahr 1921 eingeführt. in Bulgarien bestand bis 1925 kein allgemeines Wahlrecht.

Bei bikameralen parlamentarischen Systemen beziehen die Angaben sich auf das Wahlrecht zur Zweiten Kammer.

Quellen: Nohlen u. Stöver 2010 im Abgleich mit Braunias 1932. 
Die Literatur, die sich mit dem Übergang vom Mehrheits- zum Verhältniswahlrecht befasst, behandelt nun ein Ereignis, das in den meisten Fällen in den kurzen Zeitraum zwischen 1915-1920 fällt, so auch in Deutschland (vgl. Tabelle 1, siehe auch Boix 1999; Cusack et al. 2007). Natürlich existierten damals Parteien samt entsprechender Organisationen. Doch waren diese Parteien in der Regel relativ lose Vereinigungen von Wahlkomitees, die von lokalen Honoratioren geführt wurden. Dies gilt vor allem für die „etablierten“ Parteien (Nipperdey 1961; Nohlen u. Stöver 2010; Kreuzer 2001). Gerade sie spielen in den klassischen und den neueren Erklärungen der Einführung der PR aber eine entscheidende Rolle (Boix 1999, 2010; Colomer 2005; Rokkan [1970] 2009). Daraus folgt eine einfache analytische Konsequenz: Wir müssen bei diesen Parteien in der Wahlreformfrage die Interessen zweier Gruppen unterscheiden: die der Parteiführer und der Parteibasis bzw. die der Fraktionsführer und der Hinterbänkler im Parlament.

Über die Effekte des Verhältniswahlrechts auf den Legislativprozess sind wir recht gut informiert: Die Verhältniswahl fördert die Fraktionsgeschlossenheit, hauptsächlich weil Parteiführer Abgeordnete über ihren Einfluss auf die Zusammensetzung von (meist geschlossenen) Parteilisten weitreichend sanktionieren können (Carey u. Shugart 1995). Unter dem Mehrheitswahlrecht existieren solche Listen aber nicht. Für Kandidaten ist es oft noch nicht einmal zwingend, unter einem bestimmten Parteilabel anzutreten. Die Chance, gewählt zu werden, war etwa im Kaiserreich stattdessen abhängig von Ressourcen, die nicht unbedingt mit Hilfe einer Partei erlangt werden konnten, wie etwa Publizität, logistische Unterstützung oder Finanzmittel. So traten die Kandidaten damals zwar in der Regel für eine bestimmte Partei an. Aber das war nicht zwingend und wir finden in jeder Legislaturperiode etliche parteilose Reichstagsmitglieder. Auch war die jeweilige Parteiführung nicht überall und immer an einer nationalen Kampagne in allen 397 Wahlkreisen interessiert. Für sie war es oft vielversprechender (und kostengünstiger), im Gegenzug für programmatische Konzessionen des Kandidaten einer anderen Partei vor Ort, auf eine eigene Kandidatur zu verzichten oder durch gegenseitige Unterstützungsabkommen Kandidaturen miteinander zu tauschen (Reibel 2007). Auch für die Kandidaten waren solche Wahlabsprachen vorteilhaft, wenn nicht im Hinblick auf den Gewinn von Wahlkampfressourcen, dann zumindest durch die Verminderung von Konkurrenten um das Mandat.

Da diese Unterstützungsabkommen ihrer Natur nach auf einzelne Kandidaten in einzelnen Wahlkreisen zugeschnitten waren, reflektierten sie oft rein lokale Gegebenheiten und die spezifische sozio-ökonomische Struktur ihres Wahlkreises (Andeweg u. Thomassen 2005; Cain et al. 1987; Nipperdey 1993; Reibel 2011). Dieser spezifisch lokale Charakter der Wahlkreisabkommen lief aber den Versuchen der Parteiführung zuwider, kohärente Parteipositionen zu Fragen von nationaler politischer Bedeutung durchzusetzen (Cusack et al. 2007). Die Herausbildung solcher nationaler Parteipositionen wurde aber wiederum vor dem Hintergrund der Nationalisierung der Politik (Caramani 2004; Chhibber u. Kollman 1998; Kreuzer 2001) durch den Aufstieg des regulatorischen Nationalstaates und der Fundamentalpolitisierung weiter Teile der Gesellschaft (Nipperdey 1993) seit dem ausgehenden 19. Jahrhundert immer dringlicher. So geriet die Praxis des 
Mandatserwerbs in der elektoralen Arena in einen zunehmenden Gegensatz zu den Erfordernissen der Mandatsausübung in der legislativen Arena.

Mit Blick auf die Nationalisierung der Politik mussten lokale Wahlbündnisse aus Sicht der Parteieliten also schon per se immer problematischer werden. Das Problem wurde jedoch noch durch die Struktur der Parteiorganisationen verschärft. Die typische etablierte Partei des Kaiserreichs bestand zum einen aus Honoratiorenkomitees und zum andern aus Fraktionen in den Parlamenten auf den verschiedenen Ebenen des Föderalstaats. „Parteiführer“ waren in diesem System prominente oder sonst einflussreiche Parteimitglieder auf einer dieser Ebenen. Sie hatten jedoch in der Regel keine Entscheidungsmacht über die Kandidatennominierung als dem aus heutiger Sicht klassischen Machtinstrument von Parteiführungen. Denn die Nominierungen erfolgten durch hunderte autarker Wahlkreiskomitees (vgl. bereits Nipperdey 1961). Die einmal gewählten Abgeordneten waren dadurch aber für ihre Wiederwahl zuvörderst auf die Unterstützung in ihrem lokalen politischen Kontext angewiesen und gerade nicht auf ihre Parteiführung (Carey 2007). Und sie mussten umso mehr von den Zielen der eigenen Parteielite auf der nationalen Ebene abweichen, je diverser die Bündniskonstellation vor Ort war, denn in den programmatischen Unterschieden zwischen einzelnen Honoratiorengruppen lag ja gerade der Grund für die Existenz mehr als einer „etablierten“ Partei. Wenn aber im Kalkül der einzelnen Abgeordneten die lokalen und nicht die nationalen Interessen vorrangig waren, dann stand die Fraktionsgeschlossenheit als Voraussetzung für ein effektives Handeln der Parteiführungen in der legislativen Arena bei jeder einzelnen Abstimmung zur Disposition.

Je divergenter die lokalen Wahlbündnisse waren, desto problematischer musste es sein, die legislative Kohäsion innerhalb der Fraktion zu sichern. Durch den rasanten Stimmenanstieg der SPD seit den 1890er Jahren waren aber immer mehr etablierte Kandidaten gezwungen, sich zur Sicherung ihres Mandats an immer buntere Koalitionen vor Ort zu binden. Das beschriebene Spannungsverhältnis zwischen lokaler zwischenparteilicher Koordination in Wablkämpfen einerseits und nationaler innerparteilicher Kohäsion im Parlament andererseits musste dadurch zu einem immer drängenderen Problem der etablierten Parteien werden.

Anders verhielt es sich mit der SPD. Ihre Berliner Führung besaß beachtliche finanzielle und logistische Mittel, die sie frei für die Unterstützung ihr genehmer Kandidaten im Wahlkampf einsetzen konnte. Sie agierte zudem als Schiedsrichterin in Fällen, in denen in den Parteigliederungen Uneinigkeit darüber herrschte, welcher Kandidat unterstützt werden sollte. Sie besaß sogar ein Vetorecht hinsichtlich dieser lokalen Beschlüsse. Um die Jahrhundertwende hatte sie so den Nominierungsprozess im Wesentlichen monopolisiert (Nipperdey 1961; Erich u. Pikart 1966). Deshalb dient sie Michels ([1911] 1957) ja auch als Musterbeispiel für den Prozess der Herausbildung oligarchisch organisierter Parteien. Durch ihren Paria-Status gegenüber den etablierten Parteien kam die SPD zudem kaum als Partnerin für Wahlbündnisse infrage (s. unten).

Als politische Organisation war die SPD deshalb einzigartig hinsichtlich der Kandidatennominierung und folglich auch in Bezug auf das Maß an Kontrolle, das die Parteiführung über einzelne Abgeordnete ausüben konnte. Die resultierende hohe Schlagkraft der SPD als Parteiorganisation und damit auch als Parla- 
mentsfraktion markiert den Beginn genau jener neuen Ära der modernen Massenpartei, die bereits zeitgenössisch beschrieben worden ist (Neumann 1932). Bis 1918 betraf dies aber eben nur die SPD oder, entlang unseres Arguments genauer: die SPD-Parteiführung. Genau durch diesen Ausnahmestatus der SPD in Verbindung mit ihrem anwachsenden Stimmenanteil wurde der oben beschriebene, unter der MR unauflösbare Widerspruch zwischen elektoraler und parlamentarischer Handlungslogik für alle anderen Parteien immer bedrohlicher.

Zusammenfassend können wir also festhalten, dass die von Parteiführern so dringlich angestrebte Parteidisziplin im Hinblick auf die wichtigsten politischen Fragen der Zeit genau jenen zentral koordinierten Kandidatenauswahlprozess voraussetzte, der durch die lokalen Wahlabsprachen gerade verhindert wurde. In dieser Situation musste die Einführung der Verhältniswahl zu einer für die etablierten Parteiführer attraktiven Option werden, um im Konflikt mit den vielen einzelnen lokalen Nominierungsausschüssen als der „party on the ground“ (Katz u. Mair 1995) die Oberhand zu gewinnen. Umgekehrt mussten die lokal verankerten Abgeordneten an der Beibehaltung der Mehrheitswahl ein ebenso starkes Interesse haben. Solange die Einführung der PR aber eine Mehrheit im Parlament erforderte, war sie deshalb - aus Sicht unseres Arguments - unwahrscheinlich.

Es erscheint uns daher unzureichend, die Einführung der Verhältniswahl vor allem mit dem Interesse der kleineren Parteien an erhöhter Proportionalität zu erklären und zu argumentieren, dass diese Bestrebungen auf geringen Widerstand der großen Parteien getroffen seien, da diese im absoluten Mehrheitswahlrecht ja bereits dem Zwang zur Koalitionsbildung unterlegen hätten (Blais et al. 2004, S. 189, Fn. 137). Eine solche Erklärung ignoriert das erhebliche positive Interesse, das auch die (Eliten der) großen Parteien an der Einführung der PR hatten sowie den lokalen statt nationalen Charakter der Koalitionsbildung unter der MR.

Ohne Zweifel war die Mandatssicherung ein vorrangiges Ziel der Akteure. Aber dieses Ziel war durch die MR gar nicht zwingend gefährdet. Ganz im Gegenteil musste die Mehrheitswahl als geradezu verlockendes Mittel erscheinen, den politischen Gegner durch möglichst breite Blockbildung am Gewinn von Mandaten zu hindern. Sieht man somit ab von den Konsequenzen der Wahlbündnisse für das Legislativverhalten der einzelnen Parlamentarier, ergibt sich sogar eine entgegengesetzte Prognose: Die Aufrechterhaltung der MR konnte gerade im Interesse der etablierten Parteien liegen. Obwohl Cusack et al. (2010) richtigerweise die zunehmende Bedeutung zwischenparteilicher Wahlabsprachen betonen, irren sie daher, wenn sie ohne weiteres annehmen, dass Wahlabsprachen für die beteiligten Parteien langfristig elektoral problematisch geworden wären. Koordinierung unter dem Mehrheitswahlrecht gefährdete letztlich die legislativen, aber nicht die elektoralen Mehrheiten etablierter Parteien.

Wir argumentieren, dass das Problem geringer Abstimmungskohärenz der Funktionslogik des Mehrheitswahlrechts bei gegebener organisatorischer Struktur der Parteien inhärent war. Dieses Problem bestand unabhängig vom Ausmaß der elektoralen Bedrohung von Links (Rokkan 1970; Boix 1999, 2010) und wurde in Deutschland durch den Anstieg des SPD-Stimmenanteils lediglich verschärft. Die nahezu gleichzeitige Einführung der Verhältniswahl in Ländern mit unterschiedlichen Parteiensystemen, auf unterschiedlichen Niveaus der wirtschaftlichen Ent- 
wicklung und mit unterschiedlichen Formen wirtschaftlicher Organisation zeigt zudem, dass sie auch unabhängig vom Grad wirtschaftlicher „Proto-Koordination “ erfolgen konnte (Cusack et al. 2010). Die Erhöhung der Abstimmungskohärenz war hingegen typischerweise ein prominentes Motiv der Reformer (vgl. etwa Maier 1975; Mezdeg u. Nohlen 1969; Nohlen 1969, 2010; Spalinger 2003).

Mit Blick auf dieses Motiv weist unser Argument aber auf sehr geringe Erfolgsaussichten für die entsprechenden Wahlrechtsreformen auf dem Wege parlamentarischer Gesetzgebung hin. Die Möglichkeit der lokalen Absicherung des Mandats durch die einzelnen Parlamentarier als Element der MR begründet ja gerade deren Abneigung gegenüber einer Zentralisierung, wie sie mit der PR einhergeht. Wenn es zu Reformversuchen kommen sollte, dann erwarten wir aufgrund dieser Interessenlage der Abgeordneten zwar durchaus die Formulierung der PR als Zielvorstellung der Parteiführungen, aber dennoch die Beibehaltung der MR in allenfalls leicht modifizierter Form. Für die Erklärung des schlagartigen und nahezu flächendeckenden Wahlsystemwandels in Europa deutet unser Argument insofern auf die Entkopplung der Reformentscheidung vom parlamentarischen Prozess als notwendige Bedingung für die Einführung der PR hin. Im konkreten Zeitraum der Reformen (Erster Weltkrieg und unmittelbare Nachkriegszeit) mit seinen zahlreichen innenpolitischen Wirren, Verfassungsänderungen und Staatsgründungen war diese Bedingung oft erfüllt.

Im Folgenden präsentieren wir unsere empirische Evidenz für das Deutsche Kaiserreich im Zeitraum von 1890 bis 1918 . Sowohl hinsichtlich der Detailliertheit unserer Daten (z. B. mit Hinblick auf die Wahlbündnisse: alle Allianzen aller Parteien) als auch bezüglich ihrer Breite (alle Wahlen in allen Wahlkreisen) sowie Reliabilität (zwei Runden double-blind Kodierung und anschließende Gegenprüfung der Ergebnisse) haben wir größtmögliche Sorgfalt walten lassen, um den hohen Anforderungen, die zu Recht an historische Evidenz gestellt werden, gerecht zu werden (Kreuzer 2010).

\section{Die elektorale Ebene}

Bereits bei der Reichstagswahl 1890 waren Wahlbündnisse ein häufiges Phänomen und breiteten sich in den folgenden Jahren immer weiter aus. Darüber hinaus wurden die meisten Allianzen bereits vor dem ersten Wahlgang geformt und in etwaigen Stichwahlen lediglich erweitert (Abbildung 1). 
Abbildung 1: Anteil der Wahlkreise mit mindestens einem Wahlbündnis an allen Wahlkreisen, 1890-1912.

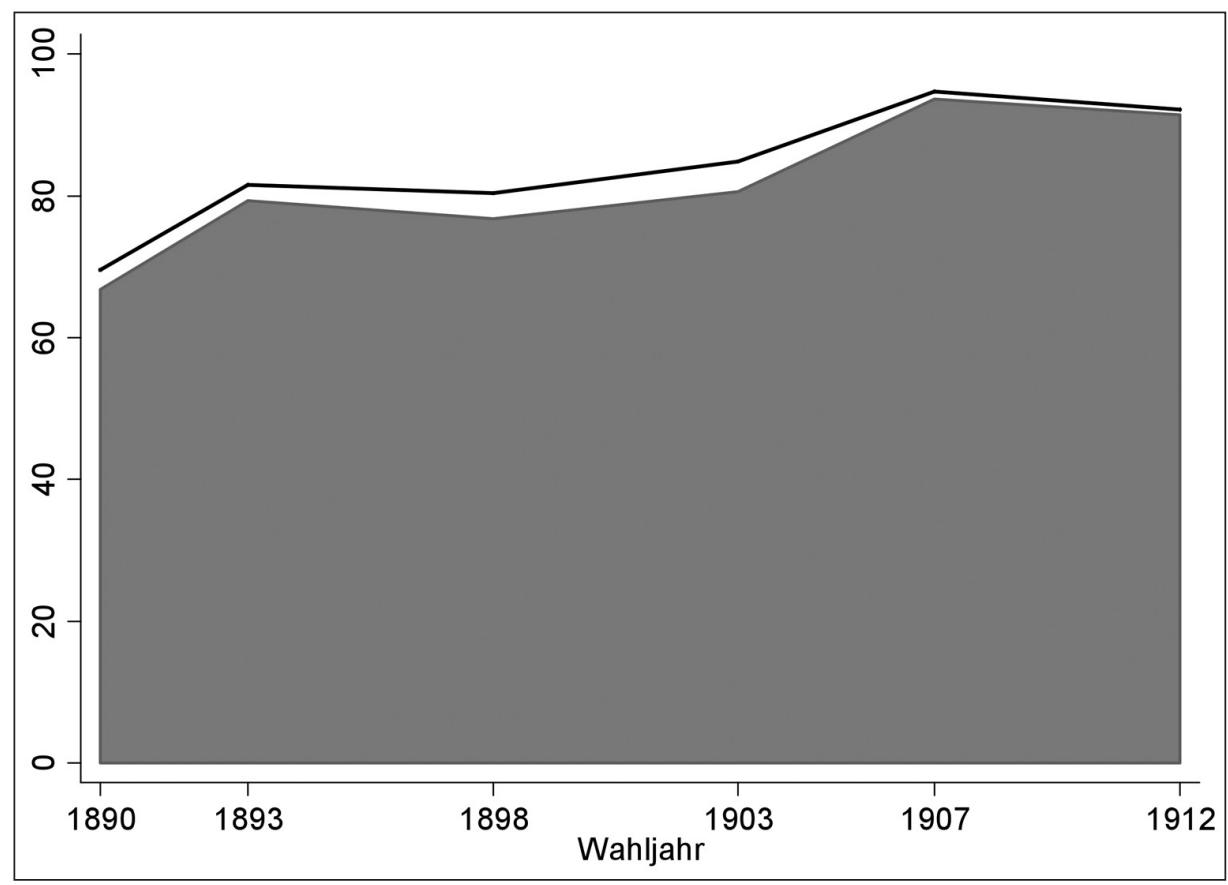

Erläuterung: Graue Fläche: Wahlbündnisse bestanden bereits zum Ersten Wahlgang; Schwarze Linie: Wahlbündnisse im Entscheidenden Wahlgang.

Quelle: Eigene Berechnungen entlang der Angaben in Reibel 2007.

Aber war es in der Tat vor allem das „etablierte“ Lager, das von ihnen Gebrauch machte? Und welche der im Reichstag vertretenen Fraktionen gehörten diesem Lager an? Als etabliert ordnen wir solche Parteien ein, deren Kandidaten überproportional von einer für Reichstagswahlen typischen Praxis profitierten: von der Unterstützung durch die Regierung. Nach Arsenschek (2003) waren das die Konservativen (DtKP, DRP) und die Nationalliberalen (NLP). Zuweilen konnte dies auch das Zentrum und die verschiedenen linksliberalen Parteien miteinbeziehen. Dieses Muster spiegelte sich auch in der legislativen Arena wider. Bei der Gesetzgebung stützte sich die Regierung vor allem auf die konservativen und nationalliberalen Fraktionen (zum parlamentarischen Verhalten der Abgeordneten im „Bündnis aus Roggen und Eisen“ als Kern legislativer Allianzen: Schonhardt-Bailey 1998). Das Zentrum und die Linksliberalen dagegen wechselten einander als Ad-Hoc-Partner dieses Kerns ab. Tabelle 2 zeigt die Verteilung der Mandate dieser parlamentarischen Lager für den Zeitraum 1890-1912. 
Tabelle 2: Mandatsverteilung im Reichstag entlang der „politischen Lager“, 1890-1912.

\begin{tabular}{lrrrrrr}
\hline & 1890 & 1893 & 1898 & 1903 & 1907 & 1912 \\
\hline Etablierte & 33,2 & 38,5 & 33,5 & 33,5 & 37,5 & 27,7 \\
Davon: Konservative & 23,2 & 25,4 & 21,7 & 20,7 & 23,7 & 16,4 \\
\multicolumn{1}{c}{ Nationalliberale } & 10,1 & 13,1 & 11,8 & 12,8 & 13,9 & 11,3 \\
Zentrum & 27,0 & 23,9 & 25,7 & 25,2 & 25,7 & 22,9 \\
Linksliberale & 20,4 & 12,3 & 13,6 & 8,8 & 13,4 & 10,6 \\
SPD & 8,8 & 11,1 & 14,1 & 20,4 & 10,8 & 27,7 \\
Ethnische Minderheiten & 6,8 & 7,3 & 6,5 & 7,1 & 7,8 & 7,1 \\
Sonstige & 3,8 & 6,8 & 6,8 & 5,0 & 4,8 & 4,0 \\
\hline Etablierte + Zentrum & 60,2 & 62,4 & 59,2 & 58,7 & 63,2 & 50,6 \\
Etablierte + Linksliberale & 53,6 & 50,8 & 47,1 & 42,3 & 50,9 & 38,3 \\
\hline
\end{tabular}

Anmerkung: Fraktionslose Abgeordnete wurden den Lagern zugeordnet, denen die Parteien ihrer Wahlbündnisse nach Reibel 2007 angehörten, wenn auch diese Parteien alle dem gleichen Lager angehörten.

Quelle: Eigene Berechnungen entlang der Angaben in Kaiserliches Statistisches Amt 189off.

Wahlbündnisse wurden nicht gleichmäßig zwischen den Parteien geschlossen. Sie waren weit überwiegend ein Instrument der liberalen und konservativen Parteien. Ihnen dienten sie geradezu als typische Strategie zur Verbesserung der Mandatschancen. So traten im Mittel gerade rund 30 Prozent der etablierten Kandidaten ohne Bündnispartner an und nur ca. 20 Prozent der gewählten etablierten Kandidaten erzielten ihr Mandat ohne solche Partner.

Diese Strategie wurde im Zeitverlauf außerdem für die etablierten Kandidaten immer gängiger. Traten 1890 noch knapp 40 Prozent von ihnen allein an, reduzierte sich deren Anteil bis 1912 auf 28 Prozent. Noch drastischer verlief die Entwicklung bei den erfolgreichen Kandidaturen. Hatten 1890 noch 36 Prozent der etablierten MdR ihr Mandat ohne Bündnispartner erlangt, stellten sie 1912 nur noch 15 Prozent der Mitglieder ihrer jeweiligen Fraktionen. Zudem wurden die Wahlbündnisse immer mehr etablierter MdR immer diverser (siehe Abbildung 2). Diese Diversität nahm bis 1907 stetig zu. Erst mit der Hinwendung der Nationalliberalen zur Fortschrittspartei bei der Wahl 1912 bricht diese Tendenz ab. Jedoch fällt die durchschnittliche Bündnisanzahl selbst bei dieser Wahl aufgrund einer Vielzahl „neuer“ konservativ-katholischer Bündnisse bezeichnenderweise nur auf das Niveau von 1903. So stützten sich die Abgeordneten etablierter Parteien auf weitaus mehr und viel heterogenere Wahlallianzen als ihre nicht-etablierten Konkurrenten. 
Abbildung 2: Verteilung der Reichstagsmandate und der Wahlbündnisse auf die Abgeordneten bzw. Kandidaten, nach politischen Lagern 1890-1912.

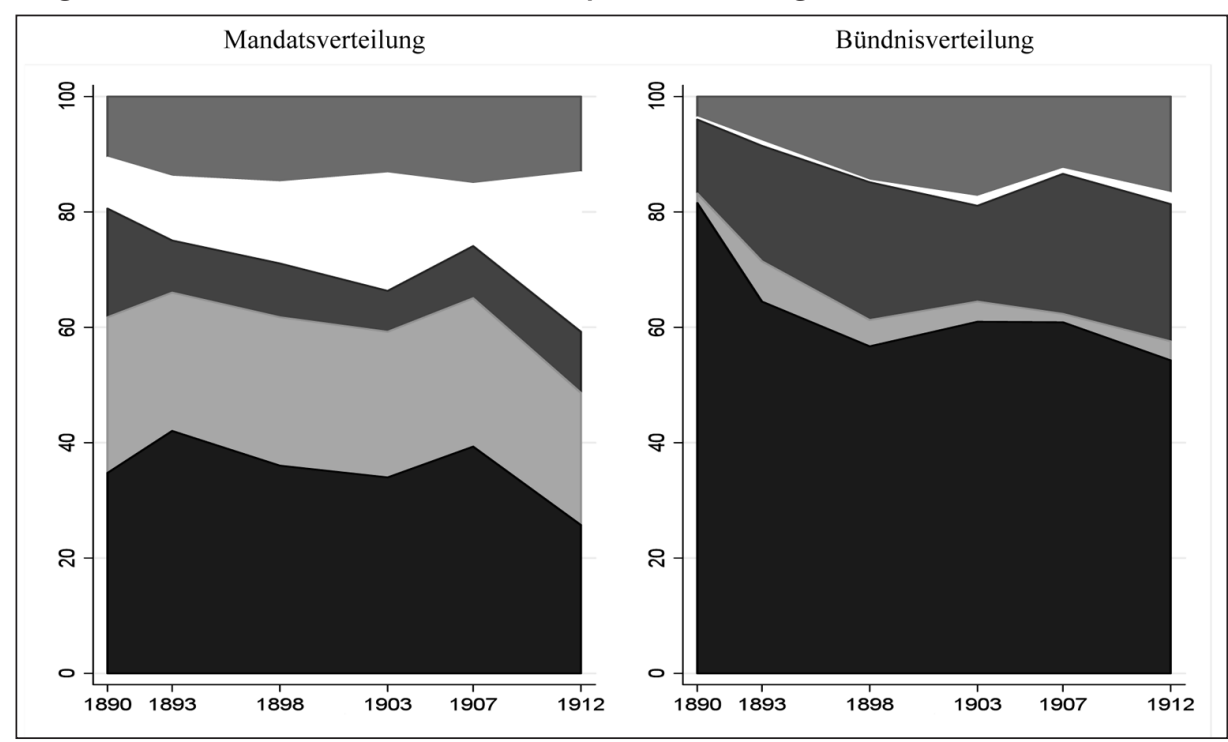

Erläuterung: Schwarz: Etablierte; Hellgrau: Zentrum; Dunkelgrau: Linksliberale; Weiß: SPD; Mittelgrau: Sonstige.

Quelle: Eigene Berechnungen entlang der Angaben in Reibel 2007.

Zudem waren die Abgeordneten der etablierten Parteien zwar nicht als einzige in lokale Absprachen eingebunden. Aber in ihren Fraktionen betraf das einen besonders großen und immer größer werdenden Anteil der Mandatsträger (siehe Abbildung 3). Nur die linksliberalen Fraktionen weisen einen vergleichbaren Anteil so eingebundener Abgeordneter auf, allerdings eben bei wesentlich geringerer Komplexität der Bündnisse, die häufig „nur“ die Zusammenarbeit der verschiedenen linksliberalen Parteien widerspiegeln. Nach der Fusion dieser Parteien zur Fortschrittlichen Volkspartei (FVP) 1909/10 treten an die Stelle dieser Partnerschaften für die Linksliberalen bei der Wahl 1912 dann Koalitionen mit den Nationalliberalen. Im starken Kontrast dazu stehen die Parlamentarier der SPD und des Zentrums: Nicht nur zog lediglich ein vergleichsweise geringer Anteil von ihnen überhaupt im Rahmen eines Wahlbündnisses in den Reichstag ein. Sondern zudem umfassten diese Bündnisse in der Regel nur einen einzigen Bündnispartner. ${ }^{2}$

2 Dies trifft auch auf die Stichwahlabkommen zu, die eine sehr ähnliche Verteilung wie in Abbildung 3 zeigen. Lediglich Kandidaten des Zentrums erhielten hier nennenswert häufiger die Unterstützung anderer Parteien; in 43 dieser insgesamt 99 Fälle von der SPD, in weiteren 23 Fällen von den Linksliberalen und in 17 Fällen von den Deutsch-Konservativen (7 davon 1912). 
Abbildung 3: Anteile der Kandidaten in Prozent, die bereits vor dem Ersten Wahlgang zusätzlich zur eigenen Partei noch von anderen Parteien unterstützt wurden, nach Parteiengruppen und Wahl 1890-1912.

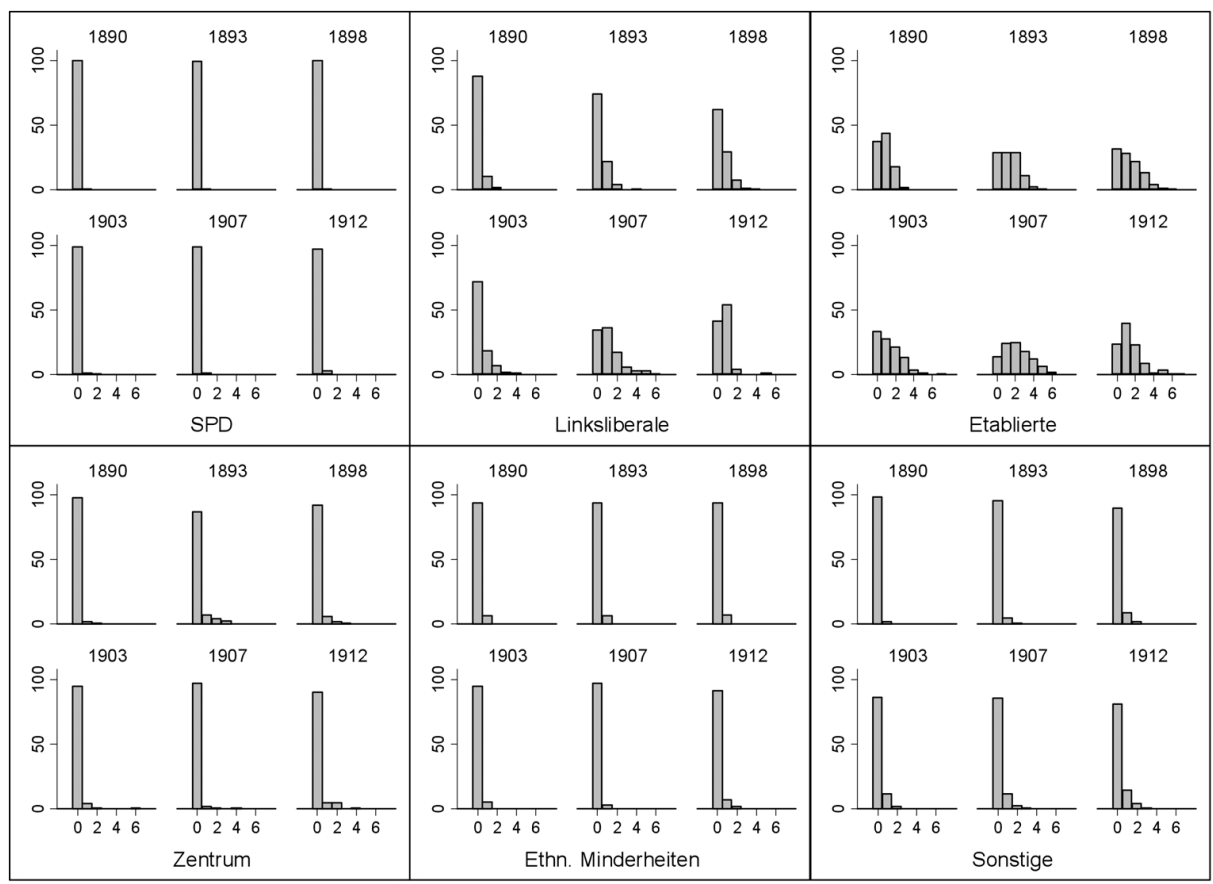

Erläuterung: Auf der X-Achse ist die Anzahl der Parteien abgetragen, von denen ein Kandidat der jeweiligen Parteiengruppe zusätzlich zu der Partei, die ihn nominierte, unterstützt wurde. Auf der YAchse wird die jeweilige Anzahl in das Verhältnis zur Gesamtzahl der Kandidaten der jeweiligen Parteiengruppe gesetzt. Bei dem Wert Null auf der X-Achse wurde ein Kandidat außer durch seine eigene Partei von keiner Partei unterstützt.

Quelle: Eigene Berechnungen entlang der Angaben in Reibel 2007.

Die Reichstagsfraktionen der etablieren Parteien waren also in ganz erheblichem $\mathrm{Maß}$ an lokale Wahlbündnisse gebunden, und zwar sowohl mit Blick auf den Anteil der betroffenen Abgeordneten als auch auf die programmatische Diversität, die von ihnen jeweils repräsentiert wurde und die das parlamentarische Verhalten jedes dieser Abgeordneten begrenzte.

\section{Die legislative Ebene}

Inwieweit wirkten sich diese lokalen Begrenzungen aber auf die legislative Arena aus? Zunächst überprüfen wir unsere Erwartung auf der Individualebene, dass aus lokalen Wahlbündnissen resultierende Zwänge es den Abgeordneten erschwerten, der Linie ihrer Fraktion im Reichstag zu folgen. In einem nächsten Schritt ermitteln wir, welche Konsequenzen dieses individuelle Verhalten für das „aggregierte“ Verhalten der Fraktionen hatte. Für diese Untersuchung nutzen wir 
einen Datensatz zum Abstimmungsverhalten jedes einzelnen MdR in der Wilhelminischen Ära. Dieser Datensatz umfasst alle namentlichen Abstimmungen (Roll Call Votes, RCV) der VIII.-XIII. Wahlperioden, beginnend im Juni 1890 und endend im Oktober 1918. Wir decken damit 217.631 Beobachtungen über das Verhalten von 1.406 Abgeordneten für einen Zeitraum von 28 Jahren ab.

Vorab ist jedoch eine kurze Bemerkung zu den Abstimmungsinhalten notwendig: Aufgrund des föderalen Charakters des Kaiserreichs war die legislative Arena nur teilweise deckungsgleich mit der nationalstaatlichen Ebene. Zentrale Bereiche staatlichen Handelns, z. B. Bildung, Kultur und öffentliche Infrastruktur, lagen in der Kompetenz der 25 Teilstaaten. Im Gegensatz dazu waren nahezu alle Aspekte wirtschaftlicher Regulierung, insbesondere des Binnen- und Außenhandels, die Geldpolitik und die Rahmensetzung im Bereich der industriellen Beziehungen, bis zum Beginn der 1890er Jahre in die Zuständigkeit des Reichs übergegangen. Nach der Einführung der Bismarckschen Sozialversicherungssysteme in den 1880er Jahren kam ein beträchtliches Arsenal redistributiver Instrumente hinzu. Wenig überraschend befasste sich denn auch ein Großteil der namentlichen Abstimmungen mit Fragen der sozio-ökonomischen Regulierung (siehe Tabelle A.1). Genau diese Fragen interessieren uns mit Blick auf unseren Bezug zur Nationalisierung der Politik, die ja gerade eine solche sozio-ökonomische Entwicklung war, besonders. Inhaltlich drängt es sich für unsere Zwecke also geradezu auf, diese Abstimmungen heranzuziehen.

Wir sind zunächst daran interessiert, in welchem Ausmaß jeder Abgeordnete dazu tendierte, von der Position seiner Partei abzuweichen. Daher haben wir jede „Parteiposition“ in jedem RCV entweder mit „Ja“, „Nein“, „Enthaltung“ oder „Abwesend“ codiert, jeweils bezogen auf das Votum der Mehrheit der Abgeordneten einer Fraktion. Anschließend haben wir Parteiposition und individuelles Stimmverhalten jedes einzelnen Abgeordneten bezogen auf seine Partei für jede Abstimmung verglichen. Stimmen, die nicht der jeweiligen Mehrheitsposition entsprachen, wurden als Fälle von innerparteilichem „Abweichen “ codiert, während solche Stimmen, die mit der Parteiposition übereinstimmten, als „Zustimmen“ gewertet wurden. Abgeordnete, die nicht an der Abstimmung teilnahmen, wurden als „Fehlend“ codiert. Unsere abhängige Variable kann folglich eine von drei Ausprägungen annehmen: Zustimmen, Abweichen und Fehlen.

Vor dem Hintergrund unseres Arguments drängt sich zunächst eine Interpretation auf, der zufolge ein Abgeordneter, dessen Kandidatur lokal auch durch eine andere Partei als seine eigene unterstützt worden war, abweichungsanfälliger sein müsste, als ein Abgeordneter, der ausschließlich für seine eigene Partei angetreten war. Mit Blick auf die Ungleichmäßigkeit von Parteiorganisationen vor Ort und politischen Strömungen vermuten wir jedoch, dass die entscheidende Variable nicht das bloße Bestehen eines Wahlbündnisses oder die Anzahl der partizipierenden Parteien waren, sondern die politische Diversität einer solchen Allianz. Diese Hypothese wendet Rokkans Vermutung zur Problematik der Wahlbündnisbildung zwischen Vertretern unterschiedlicher politischer Strömungen auf die legislative Arena an. Wir haben daher zwei Variablen für Wahlbündnisse definiert: eine Dummy-Variable, die ausdrückt, ob ein Abgeordneter von einem Wahlbündnis unterstützt wurde oder nicht („Allianz-Existenz“, 0 für nicht vorhanden und 1 für vorhanden), und drei Variablen, welche die Anzahl der in dieser Allianz vertretenen politischen Lager 
angeben (vgl. Tabelle 2), abzüglich der eigenen Partei („Bündnisvielfalt“, mit jeweils einer Dummy-Variable für ein, zwei oder drei politische Lager als Unterstützer der Partei des Abgeordneten im Wahlkreis). Als solche „Lager" klassifizieren wir, ebenfalls im Anschluss an Rokkan (1970), die SPD als Vertreterin der Arbeiter, das Zentrum für die Katholiken, die Nationalliberalen und Konservativen als Vertreter der schon erwähnten etablierten und ökonomisch protektionistischen „Hochzeit von Stahl und Roggen“, die Linksliberalen als deren verfassungspolitische und ökonomische Opponenten, die Vertreter der ethnischen Minderheiten und die Antisemiten (vgl. etwa Fairbairn 1997; Schmädeke u. Fust 1995; Sperber 1997).

Darüber hinaus sind wir daran interessiert, in welchem Maße individuelles Verhalten durch innerparteiliche Organisationsformen beeinflusst war: Inwieweit waren Parteiführer in der Lage, Abgeordneten auf die Parteilinie zu verpflichten? Um diese Frage zu beantworten, unterscheiden wir zwischen solchen Abgeordneten, die auch „Parteichefs“ waren und solchen, die es nicht waren. Anhand der Angaben in Huber (1991) und Fricke et al. (1983) haben wir all jene Abgeordnete mit 1 codiert, die entweder Vorsitzender ihrer Partei (oder, soweit einschlägig, der jeweiligen Zentralausschüsse) oder ihrer Fraktion waren. Alle anderen Abgeordneten wurden als „Hinterbänkler“ mit 0 codiert. Ist die Wahrscheinlichkeit des Abweichens für Hinterbänkler signifikant größer und für Parteiführer signifikant geringer, schließen wir darauf, dass Parteichefs die Transformation „ihrer“ Abgeordneten in einen kohärenten Stimmenblock nicht gelang. Ein solches Ergebnis ordnen wir als Resultat fehlendender organisatorischer Mittel in den Händen der Parteiführung ein. Daher haben wir zwei weitere Dummy-Variablen gebildet: eine, die identifiziert, ob ein Abgeordneter Mitglied einer anderen Fraktion als der SPD war, und eine, die Parteichefs von anderen Fraktionen als der SPD identifiziert. So können wir die Effekte des Zusammenwirkens der Eigenschaften „Parteichef“ und „Nicht-SPD-Abgeordneter" direkt untersuchen.

Wir präsentieren zwei Modelle zu den Abweichungen in Parlamentsabstimmungen (Tabelle 3). Um Verzerrungen zu vermeiden, die durch RCVs zu identischen politischen Prozessen entstehen könnten, z. B. durch mehrere Abstimmungen zum selben Tagesordnungspunkt, verwenden wir nur Daten aus der jeweils ersten Abstimmung in einer jeden Sitzung. Außerdem analysieren wir nur das Wahlverhalten jener Abgeordneten, die in Hauptwahlen (und nicht in Ersatzwahlen) gewählt wurden, die nicht ihre Fraktion wechselten und die nicht nur Hospitanten einer Fraktion waren. Unsere Ergebnisse beziehen sich somit ausschließlich auf den Kern der Fraktionen im Reichstag in einer jeden Wahlperiode. Zur Kontrolle eines möglicherweise abgeordnetenspezifischen Abstimmungsverhaltens schätzen wir robuste Standardfehler entlang der Voten eines jeden Abgeordneten als Cluster. Die Besonderheiten der sechs Legislaturperioden des gesamten Zeitraums von 28 Jahren werden zusätzlich mithilfe von Dummy-Variablen kontrolliert.

Modell I stellt die Existenz von und die Vielfalt innerhalb eines Wahlbündnisses der Wahrscheinlichkeit für individuelles Abweichen gegenüber. Außerdem analysiert es die zu erwartenden Auswirkungen der Eigenschaft als Parteichef einer anderen Fraktion als der SPD. Die Effekte von Parteiführerstatus und Mitgliedschaft in einer anderen Fraktion als der SPD für sich genommen dienen uns als Kontrollvariablen für die Schätzung des Effekts der Interaktion von beidem. 


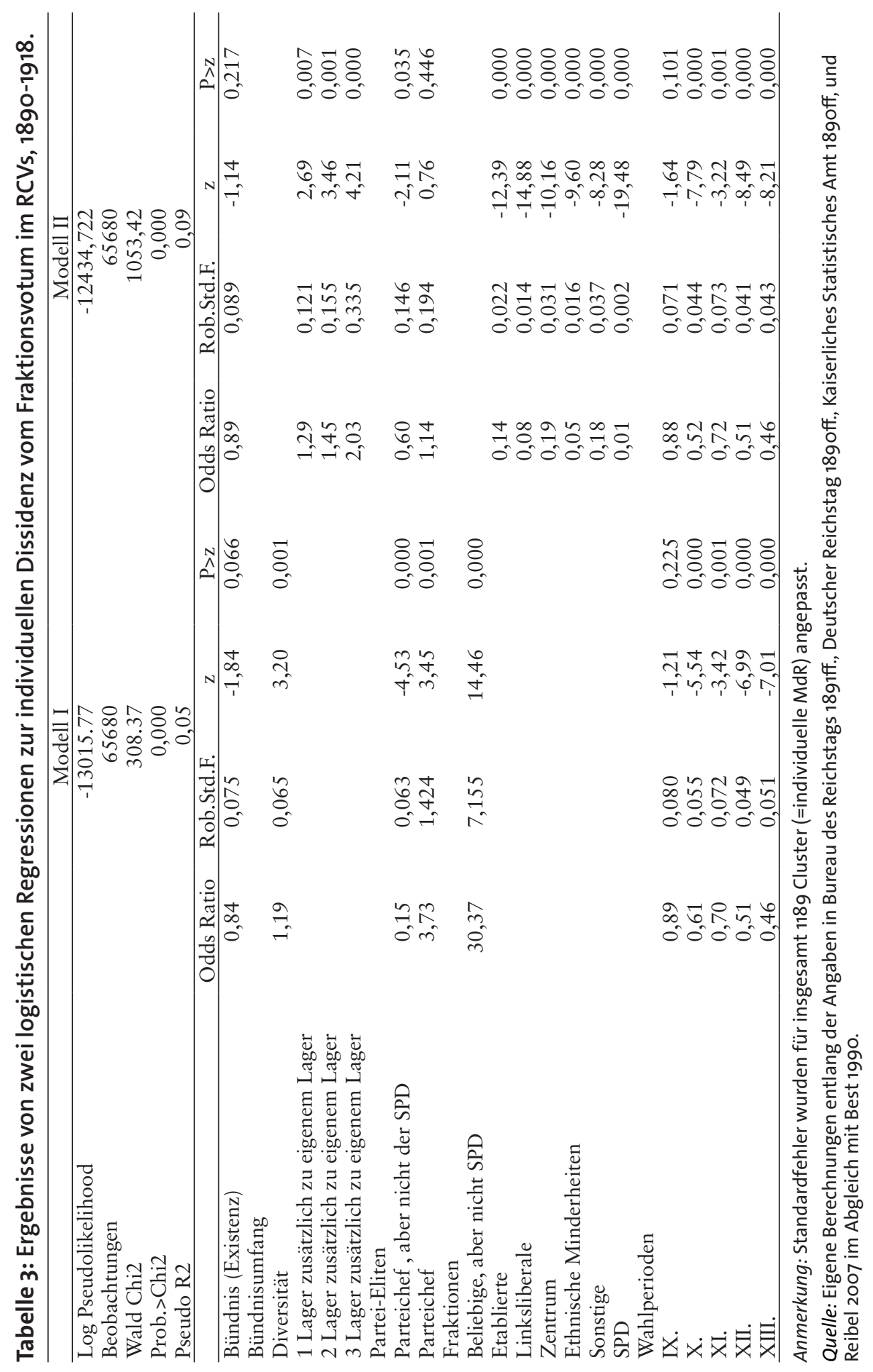


Die Ergebnisse zeigen eine statistisch hochsignifikante positive Wirkung der Vielfalt von Wahlbündnissen auf die Wahrscheinlichkeit, gegen die Linie der Partei zu stimmen. Zudem zeigt sich ein sehr deutlicher Zusammenhang zwischen der Ausprägung „Parteichef einer anderen Partei als der SPD“ und verringerter Neigung zum Abweichen. Ebenso ist das Chancenverhältnis zwischen Abweichen und $\mathrm{Zu}$ stimmen für Nicht-SPD-Abgeordnete deutlich und signifikant höher als für ihre sozialdemokratischen Kollegen. Mit den SPD-Hinterbänklern als Referenzkategorie unterschied sich das Wahlverhalten der nicht-sozialdemokratischen Parteichefs in ihrem Festhalten an der Parteilinie vergleichsweise stark von ihren eigenen Hinterbänklern. Für SPD-Parteichefs lässt sich das nicht behaupten, da die Variable Parteiführung hier keinen signifikanten Effekt zeigt (und der Koeffizient zudem ein unerwartetes Vorzeichen annimmt).

In Modell II untersuchen wir die lagerspezifischen Effekte im Detail (anstatt lediglich zwischen sozialistischen und nicht-sozialistischen Abgeordneten zu unterscheiden) und unter Berücksichtigung des genauen Grades der Diversität des jeweiligen Wahlbündnisses. In diesem Modell dienen die fraktionslosen Abgeordneten der achten Legislaturperiode als Referenzkategorie. Durch dieses Modell können wir unsere Ergebnisse erheblich verfeinern. Zunächst spielte es keine Rolle, ob ein Abgeordneter innerhalb einer Allianz gewählt wurde oder nicht, wenn wir für den Effekt der Zugehörigkeit zu einem bestimmten politischen Lager kontrollieren. Aber die Wahrscheinlichkeit, von der Parteiposition abzuweichen, steigt erheblich und kontinuierlich mit der Anzahl der im Bündnis vertretenen politischen Lager, d. h. mit der Bündnisdiversität. Fraktionsspezifische Effekte selbst sind ebenfalls sehr klar ausgeprägt. Das betrifft insbesondere die Mitgliedschaft in der SPD-Fraktion. Sie eliminiert nahezu jegliche Tendenz zu individuellem Abweichen. Für alle anderen Fraktionen ist dieser Zusammenhang zwar ebenfalls vorhanden (wie gesagt, im Vergleich zu den Fraktionslosen), jedoch wesentlich schwächer als bei der SPD. Die dortige Mitgliedschaft führte also in einem nennenswerten Maß zur Mobilisierung für die Sache der eigenen Fraktion; garantiert war sie jedoch bei Weitem nicht.

Kommen wir nun zu den Befunden im Aggregat der Parlamentsfraktionen. Unter den nicht-sozialistischen Parteien waren es vor allem die linksliberalen und etablierten Fraktionen, deren Abgeordnete zu einem großen Teil durch Allianzen über die Parteigrenzen hinweg unterstützt wurden (siehe Abbildung 4). 
Abbildung 4: Anzahl der MdR ingesamt und der MdR, die innerhalb bereits vor dem ersten Wahlgang bestehender Wahlbündnisse gewählt wurden, nach Parteiengruppen und Wahl 1890-1912.

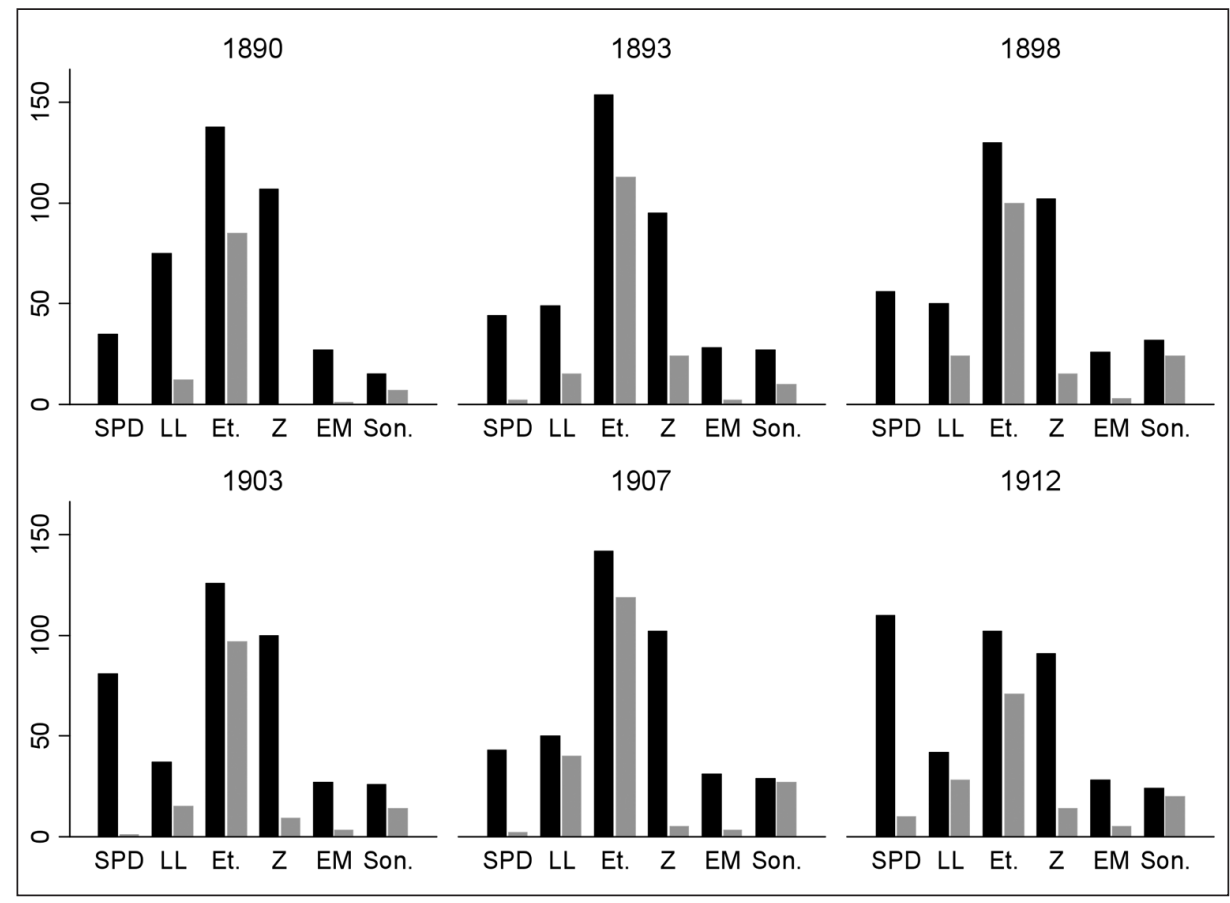

Erläuterung: Schwarz: Mandatsanzahl; Grau: Anzahl der Mandatsinhaber, die von mindestens einer weiteren Partei außer ihrer eigenen Partei unterstützt wurden.

LL: Linksliberale; Et: Etablierte; Z: Zentrum; EM: Ethnische Minderheiten; Son.: Sonstige.

Quelle: Eigene Berechnungen entlang der Angaben in Bureau des Reichstags 1891ff., Deutscher Reichstag 1890ff., Kaiserliches Statistisches Amt 189off, und Reibel 2007 im Abgleich mit Best 1990.

Die meisten dieser Bündnisse überschritten mindestens die Grenze eines politischen Lagers, und eine beträchtliche Minderheit der Abgeordneten hing von der Unterstützung von Parteien aus zwei oder mehreren politischen Lagern ab (siehe Tabelle 4). 
Tabelle 4: Anteile der Abgeordneten der politischen Lager nach der Diversität ihrer Wahlbündnisse.

\begin{tabular}{crcrrrrr}
\hline Diversität & SPD & Linksliberale & Zentrum & Etablierte & $\begin{array}{c}\text { Ethn. } \\
\text { Minderh. }\end{array}$ & Sonstige & Alle \\
\hline 0 & 84,9 & 19,8 & 79,8 & 46,6 & 88,9 & 35,2 & 59,5 \\
1 & 12,5 & 42,4 & 14,4 & 38,5 & 11,1 & 46,5 & 27,7 \\
2 & 2,7 & 27,8 & 5,3 & 12,7 & 0,0 & 16,0 & 10,6 \\
3 & 0,0 & 10,1 & 0,5 & 2,1 & 0,0 & 2,3 & 2,2 \\
\hline
\end{tabular}

Erläuterung: Diversität bezeichnet die Anzahl der in den jeweiligen Wahlbündnissen vertretenen politischen Lager außer dem eigenen Lager. Bei einer Diversität von Null kann ein Wahlbündnis zwar bestanden haben. Es umfasste dann aber nur Parteien aus dem gleichen politischen Lager. Bei SPD, Zentrum und den Parteien der ethnischen Minderheiten bedeutete eine Diversität von Null in der Regel gleichzeitig, dass überhaupt kein Bündnis zugunsten des jeweiligen Kandidaten bestand.

Quelle: Eigene Berechnungen entlang der Angaben in Bureau des Reichstags 1891ff., Deutscher Reichstag 189off., Kaiserliches Statistisches Amt 189off, und Reibel 2007 im Abgleich mit Best 1990.

Dieses Muster der Bündnisvielfalt in Verbindung mit der organisatorischen Schwäche der etablierten Parteien spiegelt sich in niedrigen Mobilisierungsraten und hohen Abweichungsquoten auf der aggregierten Ebene wider. Die einzelnen Fälle von Abweichen in den Reihen der nichtsozialistischen Parteien schwächten die legislative Schlagkraft ihrer Fraktionen erheblich. Um das zu verdeutlichen, sind in Abbildung 5 zum einen Kreise eingetragen, deren Größe dem Anteil der Mandate entspricht, den die Reichstagsfraktionen in den sechs Legislaturperioden besaßen. Auf der Vertikalen sind die Werte nach dem Rice-Index für diese Fraktionen abgetragen. Ein Wert von 1 entspricht dabei vollständiger Geschlossenheit einer Fraktion (alle Anwesenden stimmen mit der Parteiposition), ein Wert von 0 entspricht vollständiger Paralyse (gleich viele Abgeordnete einer Fraktion stimmen für und gegen die Parteiposition). Nur bei der SPD finden wir einen Grad an Geschlossenheit (um 0,98), der mit den im heutigen Bundestag (Saalfeld 1995) oder den Landtagen (Stecker 2011) erzielten Werten vergleichbar ist. Während die Rice-Werte für die Linksliberalen und Parteien der ethnischen Minderheiten (Elsässer, Dänen und Polen) noch rund 0,95 betragen, konnten die etablierten Parteien und das Zentrum kaum je Werte um 0,9 übertreffen. 
Abbildung 5: Rice-Indizes für die Reichstagsfraktionen, mit Indikation der Mandatsanteile durch verschieden große Kreise, nach Wahlperioden 1890-1918.

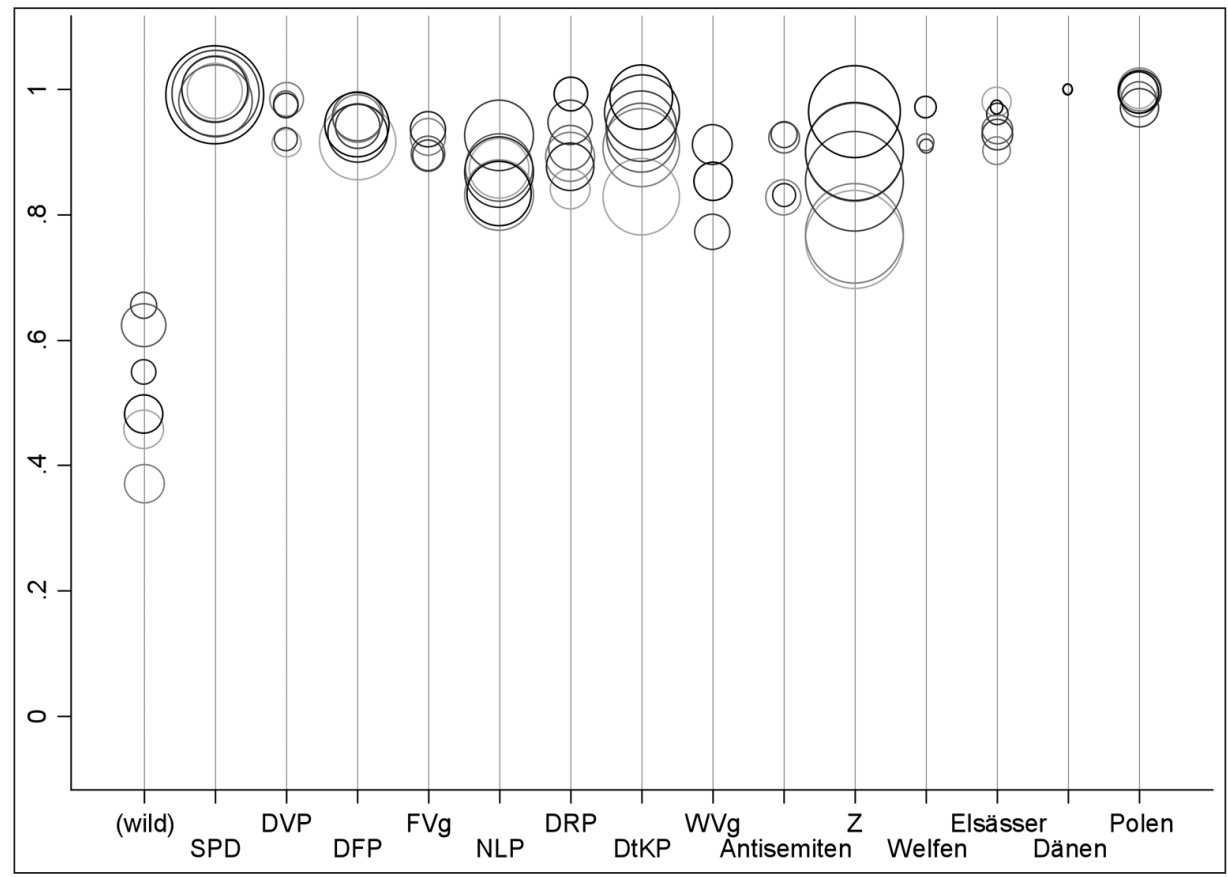

Quelle: Eigene Berechnungen entlang der Angaben in Bureau des Reichstags 1891ff., Deutscher Reichstag 189off., Kaiserliches Statistisches Amt 189off, und Reibel 2007 im Abgleich mit Best 1990.

Was bedeutete dies für die legislative Entscheidungsfindung? Solange das etablierte Lager in legislativen Koalitionen entweder mit dem Zentrum oder den Linksliberalen eine Mehrheit für seine Politik formen konnte, stellte die niedrige Geschlossenheit im Abstimmungsverhalten vielleicht in der Tat nur ein Ärgernis für die Parteiführer da. Dies galt insbesondere, da es der SPD lange Zeit nicht gelang, zu nennenswerter parlamentarischer Stärke zu gelangen. Aber nach den Wahlen von 1912 stellte sie nicht nur ein gutes Viertel aller Mandate, sondern ihre Abgeordneten wichen zudem gerade nicht von Fall zu Fall von ihrer Parteiposition ab. Zugleich bestanden bei den etablierten Parteien, den Linksliberalen und dem Zentrum niedrige Mobilisierungsraten und ein hoher Grad an innerparteilicher Dissidenz fort. Um den Effekt dieser gegensätzlichen Entwicklung auf die legislative Arena ermessen zu können, berechnen wir abschließend die „effektive legislative Stärke“ einer jeden Fraktion anstelle des reinen Anteils der Mandate. Dafür addieren wir zunächst für jede Fraktion und für jede Abstimmung alle Abgeordneten, die ihrer jeweiligen Parteiposition zustimmten. Anschließend subtrahieren wir die Zahl der abweichenden Abgeordneten, summieren die Resultate über alle Parteien auf und geben die Werte für die einzelnen Parteien als Anteilswerte dieser Summe aus. BoxPlots dieser Anteile für die legislativen Koalitionen der etablierten Parteien entweder mit den Linksliberalen oder mit dem Zentrum sind in Abbildung 6 dargestellt. 
Abbildung 6: Boxplots der mittleren Effektiven Mandatszahl für legislative Bündnisse der etablierten Parteien mit Zentrum und Linksliberalen, nach Wahlperioden 1890-1918.

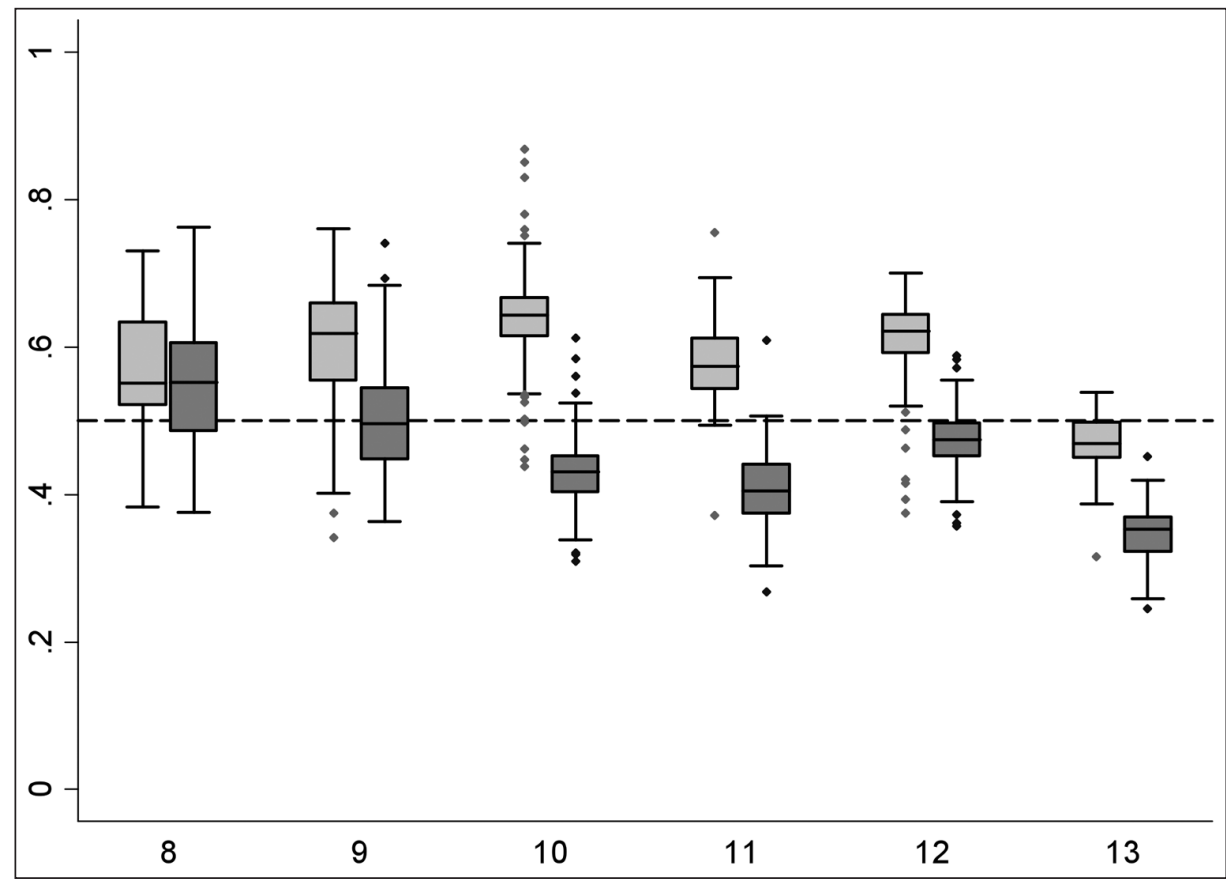

Erläuterung: X-Achse: Wahlperiode; Y-Achse: Mittelwert der Effektiven Mandatszahl; Gestrichelt: $50 \%$ der Effektiven Mandate; Hellgrau: Etablierte + Zentrum; Dunkelgrau: Etablierte + Linksliberale.

Quelle: Eigene Berechnungen entlang der Angaben in Bureau des Reichstags 1891ff., Deutscher Reichstag 189off., Kaiserliches Statistisches Amt 189off, und Reibel 2007 im Abgleich mit Best 1990.

Ein Vergleich dieser Darstellung mit den Mandatsanteilen in Tabelle 2 zeigt auf den ersten Blick keine gravierenden Unterschiede. Allerdings gibt es eine Ausnahme. Zwar gelang es den etablierten Parteien noch 1912 zumindest mit dem Zentrum eine (knappe) Mehrheit an Mandaten zu erzielen. Aber sie waren nicht mehr in der Lage, diese Mandatsmehrheit in eine legislative Mehrheit umzuwandeln. Die nicht-sozialistischen Parteien erzielten also noch immer über 60 Prozent der Reichstagssitze und noch immer bestand eine knappe Mandatsmehrheit für ein Bündnis der etablierten Parteien mit dem Zentrum. Doch unsere Ergebnisse belegen die erheblichen Probleme bei der Umsetzung von Mandatsmehrheiten in Gesetzgebungsmehrheiten für die etablierten Parteiführungen. Deshalb, und nicht wegen einer zu geringen Mandatsanzahl, war es für sie ab 1912 nicht mehr ausreichend, sich nur mit dem Zentrum oder nur mit den Linksliberalen zu koordinieren. Diese Option legislatorischer Flexibilität (wie 1898-1906 nur mit dem Zentrum und 1907-1909 nur mit den Linksliberalen) entfiel nun. Mehrheiten im Sinne der Etablierten konnten jetzt nur noch durch eine Zusammenarbeit mit beiden Gruppen gleichzeitig erzielt werden. Genau dies geschah auch, doch „war 
diese von Fall zu Fall gebildete Mehrheit von einer ,Regierungskoalition' weit entfernt" (Huber 1991, S. 324). Aufgrund der kurzen Zeitspanne bis zum Kriegsbeginn 1914 wissen wir nicht, welche Konsequenzen dies auf die Dauer für die legislativen Anliegen der etablierten Parteieliten gehabt hätte. Aber sie hätten in einem Bündnis, das praktisch alle politischen Strömungen außer den Sozialdemokraten in allen legislativen Fragen hätte umfassen müssen, umso gravierender sein müssen, je wichtiger diese Fragen für seine Teile gewesen wären.

\section{Die Wahlreform von $1917 / 18$}

Diese Befunde weisen also durchaus auf die Attraktivität einer Wahlreform für die etablierten Parteieliten hin, wenn sie entweder die Mandatszahl oder die Kohäsion ihrer Fraktionen erhöhen würde. Zu einer Reform des Reichstagswahlrechts kam es auch, und dies noch vor der in der Literatur gelegentlich einzig angeführten Einführung der Verhältniswahl durch eine Verordnung des Rats der Volksbeauftragten während der Novemberrevolution. Dabei handelt es sich um die Wahlrechtsreform von 1917/18. Auf diese Reform gehen wir nun etwas genauer ein, denn sie verlief vollständig auf dem verfassungsmäßigen Gesetzgebungsweg und damit unter den legislatorischen Normalbedingungen, unter denen die Parteivertreter vor 1918 handelten. Aufgrund der damit verbundenen ergiebigen Quellenlage lassen sich die Positionen der Beteiligten gut nachvollziehen. Vor allem ermöglicht es diese Reform aber, die Erklärungskraft unseres Arguments und die der bisherigen Erklärungen zur Einführung der PR miteinander zu vergleichen. Dieser Vergleich steht daher hier im Mittelpunkt. ${ }^{3}$ Vor dem Hintergrund unseres Arguments erwarten wir, dass dem Interesse der Parteieliten an der PR aufgrund des Widerstandes der einfachen Abgeordneten die parlamentarische Mehrheit fehlt und dass somit auf dem normalen Gesetzgebungsweg mehr als marginale Änderungen des Mehrheitswahlrechts äußerst unwahrscheinlich sind.

Die Reform des Reichstagswahlrechts war ein zentrales Element in den Bestrebungen der Reichsleitung zur „Einordnung der Arbeiterschaft in den bestehenden Staat" (Bethmann Hollweg zitiert nach Huber 1978, S. 125). Vor dem Hintergrund der krisenhafter werdenden innenpolitischen Situation seit Anfang 1917 war dafür die Berücksichtigung der Anliegen der SPD unabdingbar (Huber 1978, S. 125). Zur Wahlreform wurden dem im März 1917 gebildeten Verfassungsausschuss des Reichstags im Mai drei Vorschläge zugeleitet: je einer von SPD und USPD auf reichsweite Einführung der Verhältniswahl und einer des FVP-Parteiführers Müller-Meiningen. ${ }^{4}$ Nach dessen Vorschlag sollte die Mandatszahl der bislang unterrepräsentierten Wahlkreise erhöht und dort die Verhältniswahl eingeführt werden. Für den Rest des Reichs sollte das bisherige Mehrheitswahlsystem erhalten bleiben (Verfassungsausschuss [Reichstag] 1917).

3 In der Online-Anlage zu diesem Aufsatz geben wir zudem eine umfangreichere Darstellung mit einem Fokus auf den Positionen der einzelnen Parteivertreter.

4 Bei unserer Einordnung von Personen als „Parteiführer“ stützen wir uns auf Huber (1991) und Schiffers (1979). 
Im Ausschuss warben zunächst die Vertreter der SPD für die Verhältniswahl: „Beim Proporze habe jede Partei eine bestimmte Anzahl sicherer Wahlkreise und könne sie nach Belieben mit [...] tüchtigen Männern besetzen“ (Verfassungsausschuss [Reichstag] 1917, S. 1667). Abwägender bestand für die FVP-Vertreter „der Hauptvorzug der Verhältniswahl [...] darin, dass jeder Partei die Vertretung verschafft werden solle, auf die sie nach ihrer Wählerzahl Anspruch habe.“ Aber, so ihr Einwand: „der Einfluss der Parteileitungen werde zu sehr gestärkt“ ( Verfassungsausschuss [Reichstag] 1917, S. 1668). Auch die Vertreter der Konservativen (DtKP) monierten, durch „die Verhältniswahl, die die Macht der Parteileitungen stärke, würden zahlreiche Berufspolitiker gezüchtet" (Verfassungsausschuss [Reichstag] 1917, S. 1668). Von den Vertretern des Zentrums schließlich wurde ebenfalls „vor den Konsequenzen eines solchen Versuchs gewarnt. [...] Jede Vermehrung der Mandate sei verwerflich, die eine wesentliche Benachteiligung der bäuerlichen Bevölkerung mit sich bringe; jedenfalls dürften die süddeutschen Stimmen nicht gegenüber den norddeutschen durch die Verschiebung der Abgeordnetenzahl zurückgesetzt werden " ( Verfassungsausschuss [Reichstag] 1917, S. 1668). Schließlich gelangte der FVP-Entwurf mit den Stimmen von SPD, FVP, Zentrum und NLP in das Plenum (Huber 1991, S. 145). Er zielte auf eine Resolution des Reichstags ab, in der die Reichsleitung zur Vorlage eines Gesetzentwurfs für die Einführung der Verhältniswahl in den „großen“, d. h. bevölkerungsreichen, Wahlkreisen aufgefordert wurde.

In diesen Stellungnahmen werden zwei voneinander unabhängige Interessen der Parteivertreter deutlich. Das eine Interesse ist die Mandatsmaximierung. Konservative und Zentrum befürchteten Verschiebungen zu ihren Ungunsten. Dies hätte aber jede wahlrechtliche Veränderung mit sich gebracht. Die Ursache dafür war die seit 1869 unveränderte Wahlkreiseinteilung, die im Verlauf der Zeit zu einer starken Unterrepräsentation städtischer Wahlkreise führte, wie der Vergleich von Tabelle 5 mit Tabelle 2 zeigt. Die Situation, wie sie in Tabelle 5 geschildert wird, deutet zudem auf den deutschen Fall als genau einen solchen Fall hin, der für die Boix-Rokkanschen Erklärung der Ausgangspunkt ist: die „Bedrohung von Links“ eines zersplitterten „etablierten Lagers“. Für Zentrum und Konservative passt daher die Verweigerung gegenüber jeglicher Reform in die Mandatsmaximierungssicht bei Rokkan und Boix. Unerklärt ist es aber genau aus dieser Sicht, warum gerade die SPD die Wahlrechtsgleichheit nicht über eine Wahlkreisreform herstellen wollte, sondern durch Verhältniswahl. Denn mit Blick auf ihre Stimmenanteile (Tabelle 6) hätte sie sich durch die MR bei jeder einzelnen Wahl seit 1890 um rund 6 Prozent besser gestellt als durch die PR. Dies stellt auch die Erklärungen für die Einführung der PR in Frage, die etwa von Calvo (2009) oder Cusack et al. $(2007,2010)$ gerade für den deutschen Fall vorgetragen werden. Sie diagnostizieren für die liberalen Parteien die existenzielle Gefahr, im Mehrheitswahlrecht zwischen dem sozialistischen und dem katholischen bzw. konservativen Block zerrieben zu werden. Diese Gefahr löst sich mit Blick auf Tabellen 5 und 6 auf: Gelegentliche Unterrepräsentation um durchschnittlich rund 2,5 Prozent hätte einer beinahe ebenso großen gelegentlichen Überrepräsentation gegenüber gestanden. Problematisch an all diesen Erklärungen erscheint deshalb, dass sie einerseits auf die elektorale Arena fokussieren, aber andererseits die dortigen 
Koalitionsmöglichkeiten der Parteien übersehen. Selbst wenn von Parteien als unitarischen Akteuren ausgegangen wird, können diese Erklärungen Wahlbündnisse als Instrument gerade der kleineren und damit unter der MR vermeintlich unterlegenen Parteien so nicht erfassen, und damit letztlich auch nicht die Haltung der etablierten Parteien zu diesem Wahlsystem.

Tabelle 5: Anteil der im Reichstag durch die Mandate jeweils repräsentierten Bevölkerung entlang der „politischen Lager“, 1890-1912.

\begin{tabular}{lrrrrrr}
\hline & 1890 & 1893 & 1898 & 1903 & 1907 & 1912 \\
Etablierte & 32,0 & 36,6 & 31,2 & 28,6 & 32,1 & 23,3 \\
Davon Konservative & 21,9 & 23,3 & 19,0 & 16,6 & 19,4 & 11,8 \\
\multicolumn{1}{c}{ Nationalliberale } & 10,1 & 13,3 & 12,2 & 12,0 & 12,7 & 11,5 \\
Zentrum & 25,7 & 22,6 & 23,8 & 23,1 & 22,5 & 19,7 \\
Linksliberale & 19,5 & 11,4 & 13,2 & 7,6 & 12,8 & 8,3 \\
SPD & 13,4 & 16,6 & 20,8 & 30,4 & 21,3 & 39,0 \\
Ethnische Minderheiten & 6,2 & 6,6 & 5,5 & 6,3 & 7,7 & 6,8 \\
Sonstige & 3,2 & 6,2 & 5,5 & 4,0 & 3,6 & 2,9 \\
\hline Etablierte + Zentrum & 57,7 & 59,2 & 55,0 & 51,7 & 54,6 & 43,0 \\
Etablierte + Linksliberale & 51,5 & 48,0 & 44,4 & 36,2 & 44,9 & 31,6 \\
\hline
\end{tabular}

Quelle: Eigene Berechnungen entlang der Angaben in Kaiserliches Statistisches Amt 189off.; fraktionslose Abgeordnete wurden den Lagern zugeordnet, denen die Parteien ihrer Wahlbündnisse nach Reibel 2007 angehörten, wenn auch diese Parteien alle dem gleichen Lager angehörten.

Tabelle 6: Stimmenanteile reichsweit im ersten Wahlgang entlang der „politischen Lager“, 1890-1912

\begin{tabular}{lrrrrrr}
\hline & 1890 & 1893 & 1898 & 1903 & 1907 & 1912 \\
Etablierte & 34,9 & 31,6 & 28,1 & 27,4 & 28,1 & 25,2 \\
Davon Konservative & 18,9 & 19,0 & 15,5 & 13,4 & 13,6 & 11,6 \\
\multicolumn{1}{c}{ Nationalliberale } & 16,0 & 12,6 & 12,6 & 14,0 & 14,5 & 13,6 \\
Zentrum & 18,6 & 19,1 & 18,8 & 19,7 & 19,4 & 16,8 \\
Linksliberale & 17,9 & 10,8 & 8,6 & 6,6 & 7,8 & 12,3 \\
SPD & 19,7 & 23,3 & 27,2 & 31,7 & 28,9 & 34,8 \\
Ethnische Minderheiten & 5,0 & 4,7 & 4,8 & 5,0 & 5,1 & 4,5 \\
Sonstige & 3,9 & 10,5 & 12,3 & 9,5 & 10,6 & 6,4 \\
\hline Etablierte + Zentrum & 53,5 & 50,7 & 46,9 & 47,1 & 47,5 & 42,0 \\
Etablierte + Linksliberale & 52,8 & 42,4 & 36,7 & 34 & 35,9 & 37,5 \\
\hline
\end{tabular}

Quelle: Eigene Berechnungen entlang der Angaben in Kaiserliches Statistisches Amt 189off.; Stimmen für fraktionslose Abgeordnete wurden den Lagern zugeordnet, denen die Parteien ihrer Wahlbündnisse nach Reibel 2007 angehörten, wenn auch diese Parteien alle dem gleichen Lager angehörten.

Das andere Interesse insbesondere der Vertreter der DtKP lag darin, eine Stärkung der Parteileitungen, die sie unmittelbar mit der Verhältniswahl in Verbindung brachten, zu verhindern. Hierzu ist es für unser Argument jedoch auch wichtig nachzuvollziehen, wer genau - Parteiführer oder Hinterbänkler - diesen Punkt 
vorbrachte. Darüber geben die Berichte aus dem Verfassungsausschuss keinen Aufschluss, weil die Redner dort nicht namentlich genannt werden. Dafür ziehen wir die Plenarprotokolle heran und die Quellen zum sog. Interfraktionellen Ausschuss. Dieses informelle Gremium bestand seit Juli 1917. Dort traten die „führenden Abgeordneten “ (Matthias u. Morsey 1959, S. XXXIX) von SPD, FVP und Zentrum sowie phasenweise der NLP zusammen.

Die ersten namentlich identifizierbaren Positionen ergeben sich aus dem Protokoll der Reichstagssitzung vom 6. Juli, in welcher der zitierte Verfassungsausschussbericht behandelt wurde. Dort ordnete der FVP-Parteiführer MüllerMeiningen den Antragsinhalt als Provisorium auf dem Weg zu einer umfassenden Wahlkreisreform und „erste[n] Versuch mit der Verhältniswahl“ ein (Deutscher Reichstag 1917, S. 3508). Der konservative Hinterbänkler Kreth erklärte mit Blick auf die Verhältniswahl: „Dann können wir anderen ruhig zu Hause bleiben. Der Parteileitung wird vom Bureau [des Reichstags - die Autoren] attestiert, über wieviel Stimmen sie verfügt, und dann sitzen die Parteiführer hier zusammen, geben die Stimmen in die Urne, und unsere Debatten und alles übrige erledigt sich“ (Deutscher Reichstag 1917, S. 3514). Becker-Arnsberg (Zentrum) und List (NLP, beide Hinterbänkler und aus Wahlkreisen, die von der Reform absehbar nicht betroffen waren) akzeptierten die begrenzte Einführung der Verhältniswahl, verwahrten sich aber gegen die Ausweitung auf ganz Deutschland. Der FVP-Hinterbänkler Waldstein wiederum nannte als Einwohnerzahl, ab der ein Wahlkreis als verhältniswahltauglich einzuordnen war, die Zahl 200.000, denn die Mehrheitswahl sei nicht für die „großen Städte“ geeignet.“ ( Deutscher Reichstag 1917, S. 3514). Diese Zahl schloss 327 von 397 Wahlkreisen, in denen 245 der 287 nicht-sozialistischen Parlamentarier ihr Mandat erzielt hatten (darunter Waldstein selbst), von der Reform aus. So erfuhr die Verhältniswahl von einem FVP-Hinterbänkler den Stempel eines notwendigen Übels, von dem er selbst aber nicht betroffen gewesen wäre, während sein eigener Fraktionschefs gerade für eine Einführung der PR plädierte. Der Abgeordnete Mertin (DF-Hinterbänkler) wiederum befürwortete zwar eine „verständige Vermehrung der Wahlkreise“ (Deutscher Reichstag 1917, S. 3519), konstatierte aber auch: „Wir sind weiter der Ansicht, dass durch die Verhältniswahl ein Berufspolitikertum großgezogen werden muss, das wir als segensreich nicht ansehen können“ (Deutscher Reichstag 1917, S. 3519). Schließlich wurde der Verfassungsausschuss-Antrag mit den Stimmen von SPD, FVP, Zentrum und NLP angenommen und gelangte als Resolution mit der Aufforderung zur Erarbeitung eines Gesetzentwurfs an die Reichsleitung. Deren Entwurf (Reichsleitung 1918) gelangte schließlich im März 1918 in das Plenum und wurde dort leicht verändert im Juli 1918 verabschiedet.

Die Reform betraf insgesamt nur 47 Wahlkreise. 36 davon erhielten ein zusätzliches Mandat oder wurden mit Teilen der übrigen elf Wahlkreise zu insgesamt 26 sog. „Riesenwahlkreisen“ mit jeweils zwei bis zehn Mandaten vereinigt. Für 361 Wahlkreise änderte sich nichts. In den neuen 26 Wahlkreisen wurden insgesamt 80 Mandate durch Verhältniswahl nach d'Hondt vergeben. Listenverbindungen waren zulässig. Betroffen waren also lediglich rund 18 Prozent der nun insgesamt 441 Mandate in 7 Prozent der nun 387 Wahlkreise. Die durchschnittliche Magnitude der neuen Wahlkreise lag bei 3,1, mit einem Median bei 2. Nach Carey und 
Hix (2011, S. 393; Taagepera u. Shugart 1989; vgl. auch Auth 2006; Nohlen 2006) lässt sich das Wahlsystem in den 22 dieser Wahlkreise, in denen zwei oder drei Mandate vergeben wurden, als ein System „eigener Art“ einordnen, weil die strategischen Anreize der Wähler dort eher denen der Mehrheitswahl als der Verhältniswahl entsprechen. Das wird mit Blick auf Cox' (1997) „M+1-Regel“ deutlich. Demnach ist bei dem Mehrheitswahlsystem mit Stichwahl mit der Kandidatur von höchstens drei Parteien zu rechnen. Bezogen auf die elektorale Arena änderte sich für diese 22 Wahlkreise mit zusammen 50 Mandaten damit faktisch nichts. Nur die 30 Mandate (7 Prozent von 441) der sechs „größten“ Wahlkreise (1,6 Prozent von 387) fallen in Careys und Hix’ Kategorie der Verhältniswahl, wobei 20 Mandate in fünf dieser sechs Wahlkreise in die Subkategorie der „Verhältniswahl in kleinen Wahlkreisen“ fallen. Nur ein einziger Wahlkreis genügte mit seiner Magnitude von 10 gerade eben der Größenordnung, die für die Verhältniswahl im klassischen Sinn erforderlich ist. Die Reform bedeutete also keineswegs dass „Germany adopted proportional representation“, wie das Leemann und Mares (2014, S. 463) von ihr behaupten, oder auch nur die PR in den von der Reform betroffenen Wahlkreisen, wie Schanbacher (1982, S. 43) meint. ${ }^{5}$ Sondern sie war primär eine Mandatserhöhung für die vorher unterrepräsentierten Regionen. Dadurch adressierte sie in erster Linie das auch von der Reichsleitung konzedierte Problem der Wahlrechtsungleichheit durch die überkommene Wahlkreiseinteilung (Schanbacher 1982, S. 43).

Welche Folgen hatte die Reform für die Mandatsverteilung und für die parteiinterne Organisation? Die Regierungsvorlage enthält eine Übersicht über die Wahlergebnisse von 1903, 1907 und 1912 in den betroffenen Wahlkreisen im Zuschnitt vor und nach der Reform. Dadurch können wir ihre ungefähren mandatsmäßigen Auswirkungen simulieren, indem die tatsächlichen Wahlbündnisse als Listenverbindungen interpretiert werden. Die Ergebnisse dieser Simulation, ausgedrückt in Mandatsanteilen, finden sich in Tabelle 7. Es ergibt sich eine moderate Stärkung für die SPD und eine entsprechende Schwächung für Konservative und Zentrum. Für die Liberalen hätte sich so gut wie nichts geändert.

5 Dagegen entsprach das Wahlsystem nahezu exakt dem belgischen Wahlsystem von 1899, das in der Literatur leider gelegentlich ebenfalls als Verhältniswahlsystem identifiziert wird (vgl. z. B. Calvo 2009). Auch dort wurden die 82 durch die PR erzielbaren Mandate nach der Methode d'Hondts in Wahlkreisen mit mittleren Magnituden von 3,03 und dem Median 3 verteilt (Trefs 2010, S. 282). 
Tabelle 7: Vergleich der Mandatsverteilung 1903-1912 nach dem tatsächlichen Ergebnis und nach der Simulation für die Wahlreform 1917/18.

\begin{tabular}{lrrrrrr}
\hline & \multicolumn{3}{c}{ Mandatsanteil laut Simulation } & \multicolumn{3}{c}{$\begin{array}{c}\text { Veränderung Simulation - } \\
\text { echtes Ergebnis }\end{array}$} \\
\hline \multirow{3}{*}{ Etablierte } & 1903 & 1907 & 1912 & 1903 & 1907 & 1912 \\
Davon Konservative & 32,9 & 36,3 & 26,9 & $-0,6$ & $-1,3$ & $-0,8$ \\
$\quad 20,0$ & 21,8 & 14,7 & $-0,7$ & $-1,9$ & $-1,7$ \\
\multicolumn{1}{c}{ Nationalliberale } & 12,9 & 14,5 & 12,2 & 0,1 & 0,6 & 0,9 \\
Lentrum & 24,5 & 24,5 & 22,0 & $-0,7$ & $-1,2$ & $-0,9$ \\
Sinksliberale & 8,4 & 12,9 & 11,1 & $-0,4$ & $-0,5$ & 0,5 \\
Ethnische Minderheiten & 23,1 & 14,3 & 29,9 & 2,7 & 3,5 & 2,2 \\
Sonstige & 6,3 & 7,5 & 6,3 & $-0,8$ & $-0,3$ & $-0,8$ \\
Etablierte + Zentrum & 4,8 & 4,5 & 3,6 & $-0,2$ & $-0,3$ & $-0,4$ \\
Etablierte + Linksliberale & 41,4 & 60,8 & 48,9 & $-1,3$ & $-2,5$ & $-1,7$ \\
\hline
\end{tabular}

Quelle: Eigene Berechnungen entlang der Angaben in Reichsleitung 1918.

Interessant für unser Argument ist jedoch der Grad, zu dem die Listenwahl wirksam wurde, und damit der Effekt der Reform auf die Parteiorganisationen. Für die nichtsozialistischen Parteien wäre es in fünf (1903), sieben (1907) und zwei (1912) Wahlkreisen zum Gewinn von je zwei Mandaten gekommen (vgl .Tabelle 8). Sie gehörten zu den sechs "größten“ Wahlkreisen oder waren Hochburgen dieser Parteien. Letzteres betraf nur das Zentrum und die Polen. Die „Listen“ der nichtsozialistischen Parteien konnten also in der Regel ausgesprochen kurz sein, um alle Mandatsansprüche zu bedienen - für fast alle Parteien hätte dafür fast immer ein einziger Name genügt. Außerdem wäre es für sie jeweils nur in einem Drittel aller Wahlkreise überhaupt zum Mandatsgewinn gekommen. Das lag an den geringen Magnituden in Verbindung mit der d'Hondtschen Formel. Und alle territorial vergrößerten Wahlkreise bestanden jeweils aus einer einzigen Stadt. Nun waren gerade die liberalen Parteien und das Zentrum in der städtischen Kommunalpolitik aber in der Regel ohnehin in einheitlichen Vereinen organisiert. Deshalb wären durch die Reform bei keiner Partei besondere Änderungen der organisatorischen Strukturen notwendig geworden, schon gar nicht solche, welche die Vereinsautonomie im Verhältnis zur Macht der Parteileitungen zurückgedrängt hätten. Die Honoratioren vor Ort konnten ihre Kandidaten also genauso wie zuvor benennen. Die Möglichkeit der Listenverbindungen hätte ihnen dieses Geschäft sogar noch erleichtert, denn dadurch entfiel ja das Erfordernis, sich vor der Wahl mit den Vertretern anderer Parteien auf einen Kandidaten zu einigen. Nun hätten gegenseitige Verbindungserklärungen genügt, von denen am Ende die „Liste“ mit den meisten Stimmen profitiert hätte. Zusätzliche Mandate aus der Wahlreform hätten aber an dem Problem parlamentarischer Geschlossenheit nichts geändert. Der Widerspruch zwischen vorteilhaftem Handeln vor Ort und für die Parteiführer hätte also fortbestanden. 
Tabelle 8: Simulation der Mandatsverteilung laut Wahlreform 1917/18 in den 26 Wahlkreisen mit Magnitude >1.

\begin{tabular}{|c|c|c|c|c|c|c|c|c|c|c|c|c|}
\hline & \multicolumn{3}{|c|}{$\begin{array}{c}\text { Wahlkreise } \\
\text { ohne Mandat }\end{array}$} & \multicolumn{3}{|c|}{$\begin{array}{l}\text { Wahlkreise mit } \\
1 \text { Mandat }\end{array}$} & \multicolumn{3}{|c|}{$\begin{array}{l}\text { Wahlkreise mit } \\
>1 \text { Mandat }\end{array}$} & \multicolumn{3}{|c|}{$\begin{array}{c}\text { Mandate in Wahl- } \\
\text { kreisen mit }>1 \\
\text { Mandat }\end{array}$} \\
\hline & 1903 & 1907 & 1912 & 1903 & 1907 & 1912 & 1903 & 1907 & 1912 & 1903 & 1907 & 1912 \\
\hline Etablierte & 14 & 13 & 17 & 11 & 11 & 9 & 1 & 2 & - & 2 & 4 & - \\
\hline $\begin{array}{l}\text { Da- Konser- } \\
\text { von vative }\end{array}$ & 21 & 23 & 26 & 4 & 2 & - & 1 & 1 & - & 2 & 2 & - \\
\hline $\begin{array}{l}\text { National- } \\
\text { liberale }\end{array}$ & 19 & 16 & 17 & 7 & 9 & 9 & - & 1 & - & - & 2 & - \\
\hline Zentrum & 16 & 18 & 18 & 7 & 6 & 7 & 3 & 2 & 1 & 6 & 4 & 2 \\
\hline Linksliberale & 24 & 19 & 19 & 1 & 6 & 6 & 1 & 1 & 1 & 2 & 2 & 2 \\
\hline SPD & 3 & 3 & 2 & 8 & 12 & 11 & 15 & 11 & 13 & 41 & 31 & 41 \\
\hline $\begin{array}{l}\text { Ethn. Minder- } \\
\text { heiten }\end{array}$ & 25 & 24 & 24 & 1 & - & 2 & - & 2 & - & - & 4 & - \\
\hline Sonstige & 25 & 26 & 26 & 1 & - & - & - & - & - & - & - & - \\
\hline Gesamt & - & - & - & 8 & 11 & 12 & 18 & 15 & 14 & 53 & 49 & 45 \\
\hline
\end{tabular}

Erläuterung: „Gesamt“ gibt bei den Angaben zu den Wahlkreisen die Gesamtzahl der Wahlkreise an, auf die das Merkmal für alle Parteien zutrifft. Dadurch unterschreitet z. B. die Gesamtzahl der Wahlkreise, in denen alle Parteien 1907 jeweils genau 1 Mandat erzielten, die Zahl der Wahlkreise, in denen nur die SPD jeweils genau 1 Mandat erzielte.

Quelle: Eigene Berechnungen entlang der Angaben in Reichsleitung 1918.

Bezeichnend aus Sicht unseres Arguments ist schließlich das, was nicht geschah. Denn von den 26 Wahlkreisen grenzten zwölf an mindestens einen weiteren solchen Wahlkreis. Aus Sicht der Boix-Rokkanschen Erklärung hätte es sich für die etablierten Parteien angeboten, dort jeweils nur einen Wahlkreis einzuführen, denn durch die dann höhere Magnitude hätten sich die Nachteile aus ihrer Zersplitterung für die Mandatsverteilung reduziert. Dann wären 39 der 80 Mandate in nur zwei Wahlkreisen zu erringen gewesen. Ohne besonderen gesetzgeberischen Mehraufwand hätte es dort also tatsächlich zur Einführung der Verhältniswahl kommen können. Dieser Schritt hätte aber mit Sicherheit Änderungen in den Parteistrukturen nach sich gezogen, weil sich bei größerer Kandidatenauswahl die Koordinationsproblematik in die Parteien hinein verlagert hätte. Nur aus Sicht der Parteileitungen wäre so eine Reform wünschenswert gewesen und zwar nicht hinsichtlich der Mandatsmaximierung, sondern mit Blick auf eine Rolle als Regelungsinstanz parteiinterner Streitigkeiten über die Kandidatenaufstellung, wie sie die SPD-Parteileitung spielte. Der Verzicht auf diesen Schritt, und damit der Verzicht auf zusätzliche Mandate im Tausch für die Beibehaltung der bisherigen elektoralen Praxis, zeigt die Grenzen der legislatorischen Reformfähigkeit des Wahlsystems durch Parlamentarier, die ihr Mandat lokalen Gegebenheiten verdankten, und nicht dem Willen der nationalen Parteieliten. Die einzige Partei, die in großen Umfang mehr als ein Mandat pro Wahlkreis erzielt hätte, war die SPD. Für sie 
hätte dies aber ebenfalls keine Strukturanpassung erfordert - sie verfügte ja schon über die entsprechenden innerparteilichen Verteilungsmechanismen.

Die Wahlreform von 1917/18 bedeutete also einen Kompromiss einer breiten parlamentarischen Mehrheit entlang der jeweiligen innerparteilichen Machtverhältnisse und damit unter Berücksichtigung der innerparteilichen Strukturen. Konsequent stellte der FVP-Hinterbänkler Hoff anlässlich der vom Verfassungsausschuss gegenüber der Regierungsvorlage vorgenommenen Änderung (starre statt offener Listen) fest, dass „in der überwiegenden Mehrzahl der Wahlkreise, die praktisch für den Proporz in Frage kommen, von einer wirklichen Änderung gegenüber dem jetzigen System nicht die Rede sein kann “ (Deutscher Reichstag 1918, S. 5924). Wegen der geringen Magnituden würde „die Mehrzahl der Parteien $[\ldots]$ in dem betreffenden Wahlkreis ihren Kandidaten nominieren, wie es jetzt der Fall war" (Deutscher Reichstag 1918, S. 5925).

So entspricht die Wahlreform von 1917/18 unmittelbar der Prognose entlang unseres Arguments: Modifizierung der MR aber keine Einführung der PR, obwohl gerade dies der Wunsch der Parteieliten war.

\section{Konklusion}

Wir haben in diesem Aufsatz zwei neue und umfassende Datensätze zur elektoralen und zur legislativen Arena des Wilhelminischen Deutschland zwischen 1890 und 1918 ausgewertet. Unsere Daten zeigen die damaligen Wahlbündnissen als so weit verbreitet, dass wir sie als ein typisches Element bereits der Kandidatennominierung ansehen müssen, und nicht - wie bei den bislang allein als typisch befundenen Stichwahlbündnissen - als einen Notbehelf. Die Nominierung ist wiederum nicht nur ein zentrales Element der Aktivität in Parteien, sondern auch das Scharnier zwischen Partei und Fraktion und damit zwischen elektoraler und legislativer Arena. Die Praxis der Wahlbündnisse war deshalb für den politischen Wettbewerb unter dem Mehrheitswahlrecht konstitutiv. Doch die komplexen Anforderungen an die zwischenparteiliche Koordination auf Wahlkreisebene erschwerten zunehmend die innerparteiliche Koordination der nicht-sozialistischen Parteien im Parlament. Dies konnten wir mit den Daten zu den namentlichen Abstimmungen im Reichstag zeigen. Sie belegen das deutlich „undiszipliniertere“ Abstimmungsverhalten derjenigen Abgeordneten, die elektoral nicht nur von Parteien ihres eigenen politischen Lagers, sondern auch von lagerübergreifenden lokalen Wahlbündnissen unterstützt wurden.

Der resultierende legislative Individualismus ihrer Hinterbänkler schränkte die legislativen Möglichkeiten der Eliten etablierter Parteien in dem Maße ein, in dem Parteien parlamentarisches Gewicht gewannen, die diesem Individualismus nicht ausgesetzt waren. Von dieser Einschränkung war auch ihre Fähigkeit betroffen, das Wahlsystem selbst zu ändern, wie unsere Fallstudie zeigt. Dieser Individualismus behinderte sie aber auch dabei, ihre Parteien zu ideologisch homogenen und zu strategischem Handeln fähigen Organisationen - in der Terminologie von Katz und Mair (1995) zu Massenparteien - zu formen. Genau dieses Ziel wurde jedoch nach 1918 schnell erreicht. Straffe Organisation, Stabilisierung des Parlamentspersonals und Fraktionsdisziplin - in allen diesen Dimensionen wandelten 
sich die etablierten Parteien in Deutschland nun dramatisch. Gerade auf die etablierten Parteien - genauer: auf ihre Nachfolgerinnen DDP, DVP und DNVP - treffen die bisherigen Erklärungen über die Ausbildung elitengesteuerter innerparteilicher Strukturen, wie z. B. dauerhafte Regierungsteilnahme, Zuordnung zu einzelnen Cleavages oder feste Einbindung in ein soziales Milieu, jedoch nur begrenzt oder gar nicht zu.

Entlang unseres Arguments bestand eine zentrale Ursachen dieses raschen Wandels in der Einführung der Verhältniswahl mit ihrem System starrer Listen im November 1919. Aber diese Einführung selbst war problematisch und zwar, ebenfalls entlang unseres Arguments, innerhalb der Parteien, auf deren Strukturen sie sich auswirkte. Wir demonstrieren durch die Untersuchung der Wahlreform 1917/18 hierzu zum einen, dass den Parteivertretern - Eliten und Hinterbänklern - dieser Zusammenhang durchaus klar war. Vor allem zeigen wir aber, dass selbst die Vertreter derjenigen Parteien, die entlang der Literatur von der Einführung der PR elektoral am meisten profitiert hätten, darüber gespalten waren. Den Proponenten der Verhältniswahl aus den Parteiführungen, z. B. Erzberger, Gothein, Müller-Meiningen und Stresemann, standen Hinterbänkler gegenüber, denen gerade an ihrer Vermeidung gelegen war. Für die Wahlreform 1917/18 bedeutete diese Spaltung die Begrenzung auf einen Teil des Wahlgebiets und dessen Zerteilung in so kleine Wahlkreise, dass die starre Listenwahl für diese Parteien keinen organisatorischen Effekt mehr entfaltete.

Dies heißt nicht, dass wir im Wechsel zur Verhältniswahl den einzigen Weg zur Massenpartei sehen. Für den britischen Fall hat Cox (1987) die Herausbildung der Massenpartei aus dem Zusammenhang zwischen der durch wechselseitige Blockade der einzelnen Abgeordneten im parlamentarischen Geschäftsgang bewirkten Delegation der Initiativtätigkeit an die Regierung und damit der gegenseitigen Disziplinierung von Kabinett und (Regierungs-)Fraktion erklärt. So konnten die Parteiführer im Zeitverlauf kohärente Parteilabel „ihrer“ Partei herausbilden und die Bindung der Kandidaten an diese Label führte zur modernen Parteistruktur. Für die ebenso langfristige Herausbildung eines Systems zweier Parlamentsparteien, von denen kontinuierlich (d. h. über mindestens eine gesamte Wahlperiode) jeweils die eine die Regierung stützt und die andere nicht, wie wir es in Großbritannien beobachten, fehlten in Deutschland und in vielen weiteren westeuropäischen Staaten jedoch eine ganze Reihe von Voraussetzungen - angefangen bei der vergleichsweise rigiden Regulierung der Initiativtätigkeit im Reichstag, d. h. schon bei der Abwesenheit des Problems, vor dem sich britische Parlamentarier gestellt sahen und auf dessen Lösung Cox’ Erklärung fußt. ${ }^{6}$ Die parlamentarische Verantwortlichkeit der Regierung allein ist wiederum, wie das Beispiel der Dritten Republik in Frankreich zeigt, auch nicht hinreichend dafür, Parteien zu unitarischen Akteuren $\mathrm{zu}$ „machen“. Wollte man wiederum umgekehrt vermuten, dass die parlamentarische Verantwortlichkeit das Vorhandensein von Parteien als solchen Akteuren voraussetzt, würde man erneut mit dem britischen Fall konfrontiert werden.

6 Während im Reichstag mindestens 15 der insgesamt 397 Abgeordneten einen Antrag unterstützen mussten, damit er überhaupt eingebracht werden konnte, hatte im Unterhaus jeder Einzelne der rund 650 Mandatsträger die Möglichkeit, eine private bill einzubringen. 
Die Einführung der PR führt auch nicht zwangsläufig dazu, dass Parteien vorbildlich miteinander kooperieren. Im Gegenteil, gerade das Verhältniswahlsystem ermöglicht ja die parlamentarische Vertretung auch sehr kleinteiliger Interessen. Bei entsprechend niedrigen Repräsentationshürden verlagert sich das Problem mangelnder Kohäsion dann unter Umständen lediglich von der Fraktions- auf die Koalitionsebene. An die Stelle von programmatisch heterogenen Abgeordnetengruppen treten dann zwar programmatisch homogene Fraktionen. Aber bei ausreichend divergenter Programmatik ist kooperatives Handeln, etwa bei der Unterstützung einer Regierung, zwischen diesen nicht zwangsläufig zu erwarten. Hier leuchten bereits die Probleme auf, die in der Weimarer Republik unter der PR an die Stelle geringer fraktionsinterner Kohäsion traten.

Die Einführung der Verhältniswahl war jedoch ein Instrument, um zumindest die „Akteurswerdung“ der Parlamentsparteien zu befördern, und dies war den Zeitgenossen auch klar. Die Warnungen vor den oligarchischen Effekten der PR und der durch sie verwirklichten „Partei-Tyrannei“ spielten ja nicht umsonst eine prominente Rolle in den Reformdebatten der Zeit bis in den Weimarer Verfassungsausschuss und die verschiedenen Wahlreformversuche der 1920er Jahre hinein. Sowohl neuere als auch klassische Ansätze zur Erklärung der Einführung der Verhältniswahl haben diese entscheidende Dimension des historischen Reformprozesses jedoch bislang weitgehend ausgeblendet. Die legislativen Konsequenzen der elektoralen Anreize des Mehrheitswahlrechts spielten in der Wahlreformliteratur bislang kaum eine Rolle. Sobald diese Dimension aber berücksichtigt wird, hört das bisherige zentrale empirische Rätsel über die Einführung der Verhältniswahl - nämlich die Frage, warum die Reform zwischen den großen politischen Parteien kaum umstritten war, innerhalb der Parteien aber sehr wohl - auf, eines zu sein.

\section{Literatur}

Ahmed, Amel. 2010. Reading History Forward: The Origins of Electoral Systems in European Democracies. Comparative Political Studies 43: 1059-1088. doi: 10.1177/0010414010370436.

Ahmed, Amel. 2012. Democracy and the Politics of Electoral System Choice. Engineering Electoral Dominance. New York: Cambridge University Press.

Andeweg, Rudy B., und Jacques J. A.Thomassen. 2005. Modes of Political Representation: Towards a New Typology. Legislative Studies Quarterly 30: 507-528.

Arsenschek, Robert. 2003. Der Kampf um die Wablfreiheit im Kaiserreich: Zur parlamentarischen Wahlprüfung und politischen Realität der Reichstagswablen 1871-1914. Düsseldorf: Droste Verlag.

Auth, Pepe. 2006 De un sistema proporcional excluyente a uno incluyente. Mimeo. Santiago de Chile: Fundation Chile.

Benoit, Kenneth. 2004. Models of Electoral System Change. Electoral Studies 23: 363-389. doi: 10.1016/S0261-3794(03)00020-9.

Best, Heinrich. 1990. Abgeordnete der Reichstage des Kaiserreichs 1867/71-1918. Version: 1.0.0. Stand: 13.04.2010. GESIS. Studiennummer: ZA 8006. doi: 10.4232/1.8006. 
Blais, André, Agnieszka Dobrzynska, und Indridi H. Indridason. 2004. To Adopt or Not to Adopt Proportional Representation: The Politics of Institutional Choice. British Journal of Political Science 35: 182-190.

Boix, Carles. 1999. Setting the Rules of the Game: The Choice of Electoral Systems in Advanced Democracies. American Political Science Review 93: 609-624.

Boix, Carles. 2010. Electoral Markets, Party Strategies, and Proportional Representation. American Political Science Review 104: 404-413. doi: 10.1017/S0003055410000146.

Braunias, Karl. 1932. Das parlamentarische Wablrecht: Ein Handbuch über die Bildung der gesetzgebenden Körperschaften in Europa. Bd. 1. Berlin/Leipzig: Walter de Gruyter Verlag.

Bureau des Reichstags (Hrsg.). 1891. Amtliches Reichstags-Handbuch. Achte Legislaturperiode 1890/95. Berlin: Verlag von Trowitzsch und Sohn.

Bureau des Reichstags (Hrsg.). 1893. Nachtrag zum amtlichen Reichstags-Handbuch der für die achte Legislaturperiode 1890/95, enthaltend die Veränderungen seit dem Frühjabr 1890 bis zum 6. Februar 1893. Berlin: Verlag von Trowitzsch und Sohn.

Bureau des Reichstags (Hrsg.). 1893. Reichstags-Handbuch. Neunte Legislaturperiode 1893/98. Berlin: Verlag von Trowitzsch und Sohn.

Bureau des Reichstags (Hrsg.). 1896. Nachtrag zum amtlichen Reichstags-Handbuch für die neunte Legislatur-Periode 1893/98 nebst Mittheilungen über das neue Reichstagsgebäude. Berlin: Reichstagsdruckerei.

Bureau des Reichstags (Hrsg.). 1898. Amtliches Reichstags-Handbuch. Zehnte Legislaturperiode 1898/1903. Berlin: Reichstagsdruckerei.

Bureau des Reichstags (Hrsg.). 1902. Nachtrag zum amtlichen Reichstags-Handbuch für die 10. Legislatur-Periode 1898-1903. Berlin: Reichstagsdruckerei.

Bureau des Reichstags (Hrsg.). 1903. Amtliches Reichstags-Handbuch. Elfte Legislaturperiode 1903/1908. Berlin: Reichstagsdruckerei.

Bureau des Reichstags (Hrsg.). 1906. Nachtrag zum amtlichen Reichstags-Handbuch der 11. Legislaturperiode. Berlin: Norddeutsche Buchdruckerei und Verlagsanstalt.

Bureau des Reichstags (Hrsg.). 1907. Reichstags-Handbuch. Zwölfte Legislaturperiode. Berlin: Norddeutsche Buchdruckerei und Verlagsanstalt.

Bureau des Reichstags (Hrsg.). 1910. [1.] Nachtrag zum Reichstags-Handbuch der 12. Legislaturperiode. Berlin: Norddeutsche Buchdruckerei und Verlagsanstalt.

Bureau des Reichstags (Hrsg.). 1911. [2.] Nachtrag zum Reichstags-Handbuch der 12. Legislaturperiode. Berlin: Norddeutsche Buchdruckerei und Verlagsanstalt.

Bureau des Reichstags (Hrsg.). 1912. Reichstags-Handbuch. 13. Legislaturperiode. Berlin: Reichstagsdruckerei.

Bureau des Reichstags (Hrsg.). 1916. Nachtrag zum Reichstags-Handbuch der 13. Legislaturperiode. Berlin: Reichstagsdruckerei.

Cain, Bruce, John Ferejohn, und Morris Fiorina. 1987. The Personal Vote. Constituency Service and Electoral Independence. Cambridge, Mass.: Harvard University Press.

Calvo, Ernesto. 2009. The Competitive Road to Proportional Representation. Partisan Bias and Electoral Regime Change under Increasing Party Competition. World Politics 61: 254-295.

Caramani, Daniele. 2004. The Nationalization of Politics. The Formation of National Electorates and Party Systems in Western Europe. Cambridge: Cambridge University Press. 
Carey, John M. 2007. Competing Principals, Political Institutions, and Party Unity in Legislative Voting. American Journal of Political Science 51: 92-107.

Carey, John M. 2009. Legislative Voting and Accountability. New York: Cambridge University Press.

Carey, John M. , und Matthew Soberg Shugart. 1995. Incentives to Cultivate a Personal Vote: a Rank Ordering of Electoral Formulas. Electoral Studies 14: 417-439. doi: 10.1016/0261-3794(94)00035-2.

Carey, John M., und Simon Hix. 2011. The Electoral Sweet Spot: Low-Magnitude Proportional Electoral Systems. American Journal of Political Science 55: 383-397. doi: 10.1111/j.1540-5907.2010.00495.x.

Chhibber, Pradeep, und Ken Kollman. 1998. Party Aggregation and the Number of Parties in India and the United States. American Political Science Review 92: 329-342.

Colomer, Josep M. 2005. It's Parties that choose electoral systems (or, Duverger's laws upside down). Political Studies 53: 1-21. doi: 10.1111/j.1467-9248.2005.00514.x.

Cox, Gary W. 1987. The efficient Secret. The Cabinet and the development of Political Parties in Victorian England. New York: Cambridge University Press.

Cox, Gary W. 1997. Making Votes Count. Strategic Coordination in the World's Electoral System. New York: Cambridge University Press.

Cusack, Thomas, Torben Iversen, und David Soskice. 2007. Economic Interests and the Origins of Electoral Systems. American Political Science Review 101: 373-391.

Cusack, Thomas, Torben Iversen, und David Soskice. 2010. Coevolution of Capitalism and Political Representation: The Choice of Electoral Systems. American Political Science Review 104: 393-403. doi: 10.1017/S0003055410000134.

Deutscher Reichstag. 1890. Verzeichnis der Bevollmächtigten zum Bundesrat und der Mitglieder des Reichstags mit angehängter Fraktionsliste. Anlagen zu den Stenografischen Berichten. 8/1. Session: 1-47.

Deutscher Reichstag. 1893. Verzeichnis der Bevollmächtigten zum Bundesrat und der Mitglieder des Reichstags mit angehängter Fraktionsliste. Anlagen zu den Stenografischen Berichten. 9/1. Session: 1-48.

Deutscher Reichstag. 1894. Verzeichnis der Bevollmächtigten zum Bundesrat und der Mitglieder des Reichstags mit angehängter Fraktionsliste. Anlagen zu den Stenografischen Berichten. 9/2. Session: 1-48.

Deutscher Reichstag. 1895. Verzeichnis der Bevollmächtigten zum Bundesrat und der Mitglieder des Reichstags mit angehängter Fraktionsliste. Anlagen zu den Stenografischen Berichten. 9/3. Session: 1-47.

Deutscher Reichstag. 1896. Verzeichnis der Bevollmächtigten zum Bundesrat und der Mitglieder des Reichstags. Anlagen zu den Stenografischen Berichten. 9/4. Session: 1-47.

Deutscher Reichstag. 1898. Verzeichnis der Bevollmächtigten zum Bundesrat und der Mitglieder des Reichstags. Anlagen zu den Stenografischen Berichten. 9/5. Session: 1-47.

Deutscher Reichstag. 1899. Verzeichnis der Bevollmächtigten zum Bundesrat und der Mitglieder des Reichstags mit angehängter Fraktionsliste. Anlagen zu den Stenografischen Berichten. 10/1. Session: 1-49.

Deutscher Reichstag. 1901. Verzeichnis der Bevollmächtigten zum Bundesrat und der Mitglieder des Reichstags. Anlagen zu den Stenografischen Berichten. 10/2. Session: 1-50.

Deutscher Reichstag. 1904. Verzeichnis der Bevollmächtigten zum Bundesrat und der Mitglieder des Reichstags. Anlagen zu den Stenografischen Berichten. 11/ 1. Session: 1-54. 
Deutscher Reichstag. 1905. Verzeichnis der Bevollmächtigten zum Bundesrat und der Mitglieder des Reichstags mit angehängter Fraktionsliste. Anlagen zu den Stenografischen Berichten. 11/1. Session: 1-54.

Deutscher Reichstag. 1906. Verzeichnis der Bevollmächtigten zum Bundesrat und der Mitglieder des Reichstags mit angehängter Fraktionsliste. Anlagen zu den Stenografischen Berichten. 11/2. Session: 1-54.

Deutscher Reichstag. 1907. Verzeichnis der Bevollmächtigten zum Bundesrat und der Mitglieder des Reichstags mit angehängter Fraktionsliste. Anlagen zu den Stenografischen Berichten. 12/1. Session: 1-61.

Deutscher Reichstag. 1911. Verzeichnis der Bevollmächtigten zum Bundesrat und der Mitglieder des Reichstags mit angehängter Fraktionsliste. Anlagen zu den Stenografischen Berichten. 12/2. Session: 1-62.

Deutscher Reichstag. 1912. Verzeichnis der Bevollmächtigten zum Bundesrat und der Mitglieder des Reichstags. Anlagen zu den Stenografischen Berichten. 13/1. Session: 1-64.

Deutscher Reichstag. 1914. Verzeichnis der Bevollmächtigten zum Bundesrat und der Mitglieder des Reichstags. Anlagen zu den Stenografischen Berichten. 305: 3507-3523.

Deutscher Reichstag. 1917. 112. Sitzung am 6. Juli 1917 (Erster Bericht des 26. Ausschusses [Verfassungsausschuss]) über die Fragen der Änderung des Wahlrechts im Reiche. Stenografische Berichte über die Verhandlungen des Reichstags 13. Legislaturperiode, 2. Session: 3507-3523.

Deutscher Reichstag. 1918. 186. Sitzung am 8. Juli 1918 (Zweite Beratung des Gesetzentwurfs über die Zusammensetzung des Reichstags und die Verhältniswahl in großen Reichstagswahlkreisen). Stenografische Berichte über die Verhandlungen des Reichstags. 13. Legislaturperiode, 2. Session: 5911-5935.

Erich, Matthias, und Eberhard Pikart. 1966. Bemerkungen zur Praxis der Kandidatenaufstellung und zur Frage einer wirklich proletarischen Repräsentation, In dies.: Die Reichstagsfraktion der deutschen Sozialdemokratie 1898 bis 1918, lxvii-xc. Düsseldorf: Droste Verlag.

Fairbairn, Brett. 1997. Democracy in the Undemocratic State: The German Reichstag Elections of 1898 and 1903. Toronto: University of Toronto Press.

Fricke, Dieter, Werner Fritsch, Herbert Gottwald et al. (Hrsg.). 1983. Lexikon zur Parteiengeschichte: Die bürgerlichen und kleinbürgerlichen Parteien und Verbände in Deutschland (1789-1945). Leipzig: VEB Bibliographsches Institut.

Gourevitch, Peter. A., und James J. Shinn. 2007. Political Power and Corporate Control. The New Global Politics of Corporate Governance. Princeton: Princeton University Press.

Huber, Ernst-Rudolf. 1991. Deutsche Verfassungsgeschichte seit 1789. Bd. IV: Struktur und Krisen des Kaiserreichs. Stuttgart: Kohlhammer Verlag.

Huber, Ernst Rudolf. 1978. Deutsche Verfassungsgeschichte seit 1789. Bd. V: Weltkrieg, Revolution und Reichserneuerung. Stuttgart: Kohlhammer Verlag.

Iversen, Torben, und David Soskice. 2006. Electoral Institutions and the Politics of Coalitions: Why Some Democracies Redistribute More Than Others. American Political Science Review 100: 165-181.

Kaiserliches Statistisches Amt. 1890. Die allgemeinen Wahlen für die achte LegislaturPeriode des Reichstags im Jahre 1890. Monatshefte zur Statistik des Deutschen Reiches 1893: 24-69. 
Kaiserliches Statistisches Amt. 1893. Statistik der Reichstagswahlen von 1893 (Neunte Legislatur-Periode). Vierteljahreshefte zur Statistik des Deutschen Reiches 2: 1-55.

Kaiserliches Statistisches Amt. 1903. Vergleichende Übersicht der Reichstagswahlen von 1898 und 1903. Vierteljabreshefte zur Statistik des Deutschen Reiches 12: 41-107.

Kaiserliches Statistisches Amt. 1904. Allgemeine Statistik der Reichstagswahlen von 1903. Vierteljabreshefte zur Statistik des Deutschen Reiches 12: 2-60.

Kaiserliches Statistisches Amt. 1913. Vergleichende Übersicht der Reichstagswahlen von 1907 und 1912. Statistik des Deutschen Reichs 250: 1-77.

Kaiserliches Statistisches Amt. 1913. Die Reichstagswahlen von 1912. Statistik des Deutschen Reichs 250: 1-141. Katz, Richard S., und Peter Mair. 1995. Changing Models of Party Organizations and Party Democracy. The Emergence of the Cartel Party. Party Politics Volume 1, Heft 1: 5-28. doi: 10.1177/1354068895001001001.

Kreuzer, Marcus. 2001. Institutions and Innovation: Voters, Parties, and Interest Groups in the Consolidation of Democracy - France and Germany, 1870-1939. Ann Arbor: University of Michigan Press.

Kreuzer, Marcus. 2010. Historical Knowledge and Quantitative Analysis: The Case of the Origins of Proportional Representation. American Political Science Review 104: 369-392.

Leemann, Lucas, und Isabela Mares. 2014. The Adoption of Proportional Representation: One Phenomenon, a Plethora of Explanations. Journal of Politics 76: 461-478.

Lehmann, Sibylle H. 2010. Chaotic shop-talk or efficient parliament? The Reichstag, the parties, and the problem of governmental instability in the Weimar Republic. Public Choice 144: 83-104. doi: 10.1007/s11127-009-9505-0.

Maier, Charles S. 1975. Recasting Bourgeois Europe: Stabilization in France, Germany, and Italy in the Decade after World War I. Princeton: Princeton University Press.

Matthias, Erich, und Rudolf Morsey. 1959. Einleitung. In dies.: Der Interfraktionelle Ausschuss 1917/18, XI-LXXI. Düsseldorf: Droste Verlag.

Mezdeg, Gabriele, und Dieter Nohlen. 1969. Frankreich. In Die Wabl der Parlamente und anderer Staatsorgane, Hrsg. Dolf Sternberger, Bernhard Vogel, Dieter Nohlen und Klaus Landfried. 441-554. Berlin: Walter de Gruyter Verlag.

Michels, Robert. [1911] 1957. Zur Soziologie des Parteiwesens in der moderen Demokratie: Untersuchungen über die oligarchischen Tendenzen des Gruppenlebens. 2. Aufl. Stuttgart: Kröner.

Neumann, Sigmund. 1932. Die politischen Parteien in Deutschland. Berlin: Junker und Dünnhaupt.

Nipperdey, Thomas. 1961. Die Organisation der deutschen Parteien vor 1918. Düsseldorf: Droste Verlag.

Nipperdey, Thomas. 1993. Deutsche Geschichte, 1866-1918, Bd. II: Machtstaat vor der Demokratie. München: Verlag C. H. Beck.

Nohlen, Dieter. 1969. Italien. In Die Wahl der Parlamente und anderer Staatsorgane, Hrsg. Dolf Sternberger, Bernhard Vogel, Dieter Nohlen und Klaus Landfried, 713-752. Berlin: Walter de Gruyter Verlag.

Nohlen, Dieter. 2006. La reforma del sistema binominal desde una perspective comparada. Reviste de Ciencia Politica 26: 191-202.

Nohlen, Dieter. 2010. France. In Elections in Europe: A Data Handbook. Hrsg. Dieter Nohlen und Philip Stöver, 639-722. Baden-Baden: Nomos Verlag. 
Nohlen, Dieter, und Stöver, Philip (Hrsg.). 2010. Elections in Europe: A Data Handbook. Baden-Baden: Nomos Verlag.

Powell, G. Bingham. 2000. Elections as Instruments of Democracy. Majoritarian and Proportional Visions. New Haven/London: Yale University Press.

Reibel, Carl-Wilhelm. 2007. Handbuch der Reichstagswablen 1890-1918: Bündnisse, Ergebnisse, Kandidaten. Düsseldorf: Droste Verlag.

Reibel, Carl-Wilhelm. 2011. Bündnis und Kompromiss: Parteienkooperation im Deutschen Kaiserreich 1890-1918. Historische Zeitschrift 293: 71-114.

Reichsleitung. 1918. Entwurf eines Gesetzes über die Zusammensetzung des Reichstags und die Verhältniswahl in großen Reichstagswahlkreisen. Anlagen zu den Stenografischen Berichten 323: 1-124.

Rokkan, Stein. 1970. Citizens, Elections, Parties: Approaches to the Comparative Study to the Process of Development. Oslo: Universitetsforlaget.

Rokkan, Stein. [1970] 2009. Citizens, Elections, Parties: Approaches to the Comparative Study to the Process of Development. Colchester: ECPR Press.

Saalfeld, Thomas. 1995. Parteisoldaten und Rebellen: Eine Untersuchung zur Geschlossenheit der Fraktionen im Deutschen Bundestag (1949-1990). Opladen: Leske + Budrich.

Schanbacher, Eberhard. 1982. Parlamentarische Wablen und Wablsystem in der Weimarer Republik: Wablgesetzgebung und Wablreform im Reich und in den Ländern. Düsseldorf: Droste Verlag.

Schiffers, Reinhard. 1979. Der Hauptausschuss des Deutschen Reichstags 1915-1918: Formen und Bereiche der Kooperation zwischen Parlament und Regierung. Düsseldorf: Droste Verlag.

Schmädeke, Jürgen, und Brigitte Fust. 1995. Wählerbewegung im Wilhelminischen Deutschland - Die Reichstagswablen von 1890 bis 1912: Wablergebnisse und Strukturen im Kartenbild. Akademie-Verlag.

Schonhardt-Bailey, Cheryl. 1998. Parties und Interests in the 'Marriage of Iron and Rye'. British Journal of Political Science 28: 291-332.

Spalinger, Andrea. 2003. Die Proporzbewegung während der Dritten Republik Frankreichs. Zürich: Schulthess Verlag.

Sperber, Jonathan. 1997. The Kaiser's Voters. Electors and Elections in Imperial Germany. Cambridge: Cambridge University Press.

Stecker, Christian. 2011. Bedingungsfaktoren der Fraktionsgeschlossenheit: Eine vergleichende Analyse der deutschen Länderparlamente. Politische Vierteljahresschrift 52: 424-447.

Taagepera, Rein, und Matthew S. Shugart. 1989. Seats and Votes: The Effects and Deteminants of Electoral Systems. New Haven: Yale University Press.

Trefs, Matthias. 2010. Belgium. In Elections in Europe: A Data Handbook. Hrsg. Dieter Nohlen und Philip Stöver, 267-317. Baden-Baden: Nomos Verlag

Verfassungsausschuss (Reichstag). 1917. Erster Bericht des Verfassungssausschusses: Teilbericht über die Fragen der Änderung des Wahlrechts im Reiche. Anlagen zu den Stenografischen Berichten 321: 1666-1692. 


\section{Autorenangaben}

Dr. Valentin Schröder, Universität Bremen, Zentrum für Sozialpolitik, Mary-Somerville-Straße 5, 28359 Bremen, schroder@uni-bremen.de

Prof. Dr. Philip Manow

Universität Bremen, Zentrum für Sozialpolitik, Mary-Somerville-Straße 5, 28359 Bremen,

manow@zes.uni-bremen.de 
Anhang: Politikfelder der namentlichen Reichstagsabstimmungen 1890-1918

Tabelle A.1: Namentliche Abstimmungen im Reichstag, nach Politikfeld 1890-1918.

\begin{tabular}{|c|c|c|c|c|}
\hline \multirow[t]{2}{*}{ Politikfeld } & \multicolumn{2}{|c|}{ Insgesamt } & \multicolumn{2}{|c|}{$\begin{array}{l}\text { Ohne nachfolgende } \\
\text { Abstimmungen am } \\
\text { gleichen Tag zum gleichen } \\
\text { Tagesordnungspunkt } \\
\end{array}$} \\
\hline & $\mathrm{N}$ & $\%$ & $\mathrm{~N}$ & $\%$ \\
\hline Rüstung & 36 & 6,5 & 27 & 9,6 \\
\hline Reichshaushalt & 23 & 4,2 & 18 & 6,4 \\
\hline Binnenhandel und Handelsrecht i. w. S. & 37 & 6,7 & 22 & 7,8 \\
\hline Ethnische Minderheiten & 17 & 3,1 & 10 & 3,6 \\
\hline Außenhandel & 151 & 27,3 & 49 & 17,4 \\
\hline Mandatsprüfung & 20 & 3,6 & 15 & 5,3 \\
\hline Infrastruktur & 1 & 0,2 & 1 & 0,4 \\
\hline Arbeits- und Betriebsstättenrecht & 11 & 2,0 & 9 & 3,2 \\
\hline Geldpolitik & 2 & 0,4 & 2 & 0,7 \\
\hline Strafrecht & 35 & 6,3 & 13 & 4,6 \\
\hline Geschäftsordnung des Reichstags* & 22 & 4,0 & 10 & 3,6 \\
\hline Religion, religiöse Freiheit & 7 & 1,3 & 6 & 2,1 \\
\hline Sozialversicherung & 32 & 5,8 & 17 & 6,0 \\
\hline Steuern & 80 & 14,5 & 37 & 13,1 \\
\hline Verfassung & 17 & 3,1 & 10 & 3,6 \\
\hline Beamte, Öffentlicher Dienst & 6 & 1,1 & 1 & 0,4 \\
\hline Öffentliches und Verwaltungsrecht & 16 & 2,9 & 5 & 1,8 \\
\hline Kolonien & 5 & 0,9 & 3 & 1,1 \\
\hline Finanzmarktregulierung & 6 & 1,1 & 4 & 1,4 \\
\hline Verbraucherschutz & 9 & 1,6 & 8 & 2,8 \\
\hline Agrarpolitik (Redistributive Aspekte) & 11 & 2,0 & 8 & 2,8 \\
\hline Zivilrecht & 9 & 1,6 & 7 & 2,5 \\
\hline Insgesamt & 553 & 100,0 & 282 & 100,0 \\
\hline
\end{tabular}

*Interpretation oder Änderung der Geschäftsordnung des Reichstags (nicht: im Verweis auf die Geschäftsordnung vorgenommene Versuche, durch Namentliche Abstimmungen das Gesetzgebungsverfahren aufzuhalten [„Filibuster"] oder zu beschleunigen; diese Abstimmungen wurden bei dem jeweiligen Politikfeld aufgenommen).

Quelle: Eigene Berechnungen entlang der Angaben auf www.Reichstagsprotokolle.de; zuletzt eingesehen am: Zugegriffen: 25.6.2014. 